\title{
The Balance of Excitatory and Inhibitory Synaptic Inputs for Coding Sound Location
}

\author{
Munenori Ono and Douglas L. Oliver \\ Department of Neuroscience, University of Connecticut Health Center, Farmington, Connecticut 06030-3401
}

The localization of high-frequency sounds in the horizontal plane uses an interaural-level difference (ILD) cue, yet little is known about the synaptic mechanisms that underlie processing this cue in the inferior colliculus (IC) of mouse. Here, we study the synaptic currents that process ILD in vivo and use stimuli in which ILD varies around a constant average binaural level (ABL) to approximate sounds on the horizontal plane. Monaural stimulation in either ear produced EPSCs and IPSCs in most neurons. The temporal properties of monaural responses were well matched, suggesting connected functional zones with matched inputs. The EPSCs had three patterns in response to ABL stimuli, preference for the sound field with the highest level stimulus: (1) contralateral; (2) bilateral highly lateralized; or (3) at the center near 0 ILD. EPSCs and IPSCs were well correlated except in center-preferred neurons. Summation of the monaural EPSCs predicted the binaural excitatory response but less well than the summation of monaural IPSCs. Binaural EPSCs often showed a nonlinearity that strengthened the response to specific ILDs. Extracellular spike and intracellular current recordings from the same neuron showed that the ILD tuning of the spikes was sharper than that of the EPSCs. Thus, in the IC, balanced excitatory and inhibitory inputs may be a general feature of synaptic coding for many types of sound processing.

Key words: auditory pathways; inferior colliculus; interaural intensity difference; mouse; sound localization; synaptic current

\section{Introduction}

The balance of excitatory and inhibitory synaptic inputs is important for information processing in the CNS (Burger and Pollak, 2001; Wehr and Zador, 2003; Sun et al., 2010). For example, the relationship of the excitatory to the inhibitory current is critical for gain control in the inferior colliculus (IC) in the midbrain auditory system (Sivaramakrishnan et al., 2004; Xiong et al., 2013). The IC has unique neural circuits to supply excitation and inhibition to its neurons because it receives multiple, parallel excitatory and inhibitory inputs from lower auditory brainstem centers (Oliver, 2000; Burger and Pollak, 2001; Ito and Oliver, 2010; Malmierca and Hackett, 2010). These inputs determine, in part, the information transmitted from the IC to the auditory cortex.

Processing information about sound location is an important function of the IC (Kuwada et al., 1997), and, in this processing, the balance between excitatory-inhibitory inputs may be critical. Many IC neurons are excited by sounds in the contralateral ear and inhibited by sounds to the ipsilateral ear (re: recording site). This "EI" response is traditionally associated with sensitivity to interaural-level differences (ILDs), the primary cue for the local-

\footnotetext{
Received July 10, 2013; revised Jan. 11, 2014; accepted Feb. 3, 2014.

Author contributions: M.0. and D.L.0. designed research;M.0. performed research; M.0. analyzed data; M.0. and D.L.O. wrote the paper.

This work was funded by National Institute on Deafness and Other Communication Disorders Grant R01 DC000189. We thank Duck Kim and Charles Liberman for advice on the cochlear microphonic recordings and Shig Kuwada for critical reading of this manuscript.

Correspondence should be addressed to Dr. Douglas L. Oliver, Department of Neuroscience, University of Connecticut Health Center, Farmington, CT 06030-3401. E-mail: doliver@neuron.uchc.edu.

DOI:10.1523/JNEUROSCI.2954-13.2014

Copyright $\odot 2014$ the authors $\quad 0270-6474 / 14 / 343779-14 \$ 15.00 / 0$
}

ization of high-frequency sounds, the primary sound localization cue in mice (Lauer et al., 2011). ILD sensitivity in IC neurons is believed to be attributable to the inputs from lateral superior olive (LSO). However, ILD in the IC was shown to be modulated by pharmacological blocking of inhibitory receptors in the IC (Park and Pollak, 1994), and this suggests a complex interaction of excitation and inhibition within the IC (Li et al., 2010; Li and Pollak, 2013). Despite the importance of the ILD cue, little is known about the synaptic response to changes in interaural level in realistic scenarios such as when a sound source moves across the azimuth. Moreover, there are few direct measurements of separate excitatory and inhibitory synaptic currents involved in any type of ILD coding in the auditory system (Xiong et al., 2013). Other intracellular studies of binaural responses have not used voltage clamp to isolate the synaptic currents from the postsynaptic voltage-gated conductances (Kuwada et al., 1997; Li et al., 2010; Li and Pollak, 2013).

Here, we used whole-cell recordings to study the synaptic responses of IC neurons to binaural sounds in the intact mouse. We investigated whether excitatory and inhibitory synaptic inputs were driven by monaural or binaural stimulation in which the level at the two ears was varied around a fixed base level [average binaural level (ABL); Irvine, 1987; Semple and Kitzes, 1987] to mimic the changes in ILD that occur when a sound source is located at different points along the horizontal plane. We also asked whether excitatory and inhibitory synaptic responses to binaural stimuli were predicted from the summed responses to monaural stimuli and whether this prediction was equal for EPSCs and IPSCs. Finally, in a small number of neurons, we successfully recorded the same neuron in cell-attached and 
A

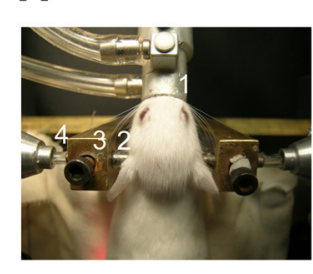

B

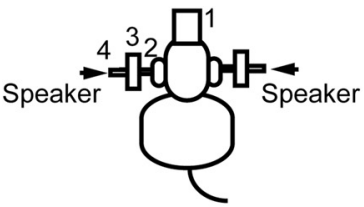

1. Nose piece

2. Metal tube for sound delivery

3. Supporter for metal tube

4. Sylycon tube

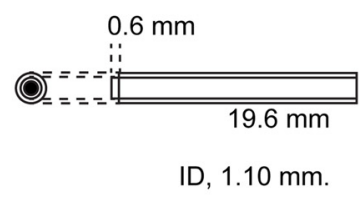

OD1, $1.67 \mathrm{~mm}$

OD2, $2.41 \mathrm{~mm}$
C
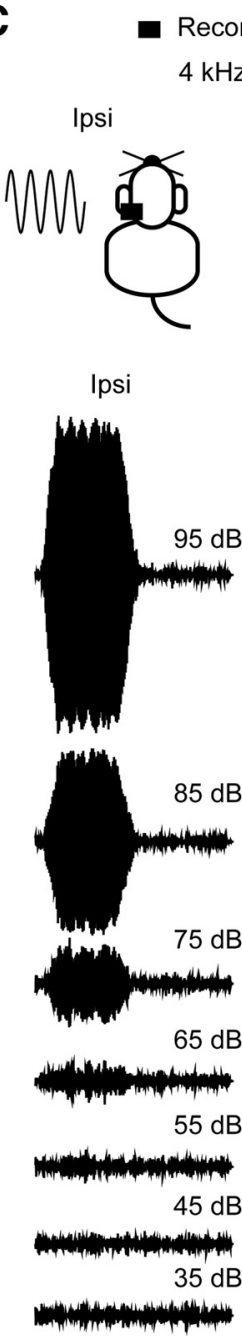

E

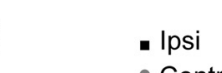

Recording side

$4 \mathrm{kHz}$ tone

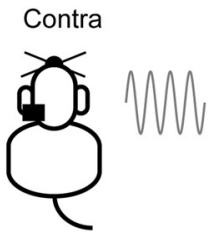

Contra

$110 \mathrm{~dB}$
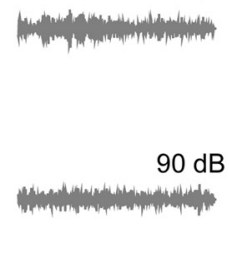

\section{$\mathrm{dB}$}

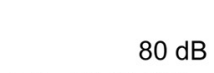

Whing

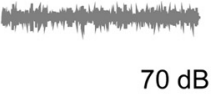

4

$60 \mathrm{~dB}$

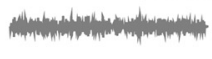

\section{.}
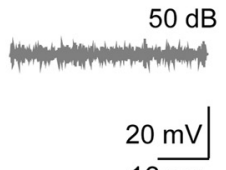

$10 \mathrm{~ms}$
D

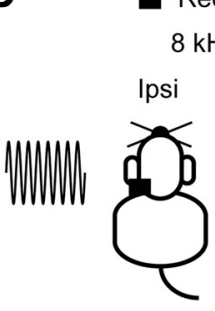

Ipsi

Recording side

$8 \mathrm{kHz}$ tone

Ipsi

Contra

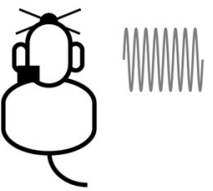

Contra

$115 \mathrm{~dB}$

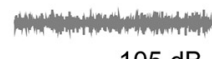

$105 \mathrm{~dB}$
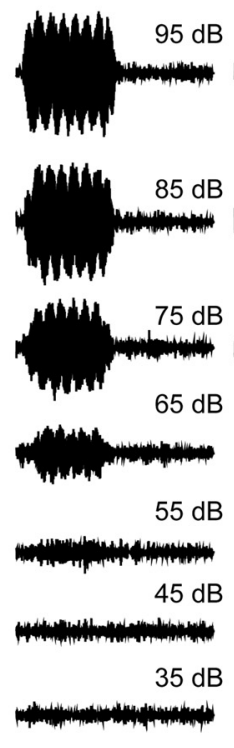

$\mathbf{F}$
$75 \mathrm{~dB}$

$65 \mathrm{~dB}$

$95 \mathrm{~dB}$

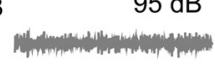

$\mathrm{dB}$

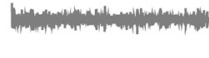

$5 \mathrm{~dB}$

$35 \mathrm{~dB}$
I Baseline with $3 \mathrm{SD}$

- Attenuation level
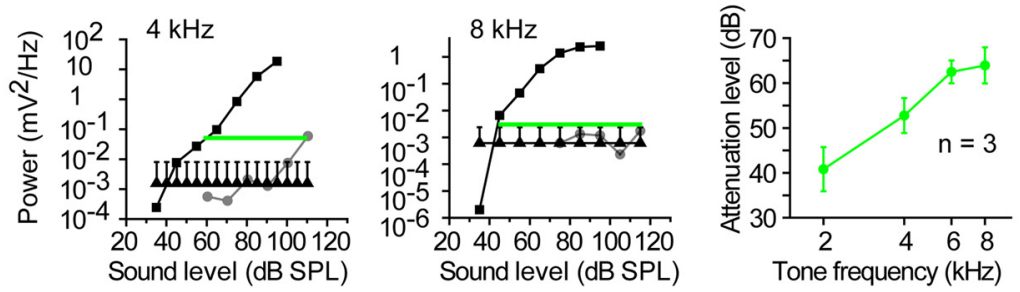

Figure 1. The binaural closed sound system for mice. $A$, Left, A photograph of the closed sound system. Right, A schematic drawing of the closed sound system. $\boldsymbol{B}$, A dimensional drawing of the metal tube for sound delivery. The tube consists of two metal tubes with different diameters. ID, Inner diameter; $0 D$, outer diameter. $\mathbf{C}-\boldsymbol{F}$, The measurement of the binaural acoustic crosstalk. $C, D, C M$ responses to ipsilateral and contralateral tone stimuli. Left traces are the responses to ipsilateral stimuli, and right traces are the responses to contralateral stimuli. The sound level is shown with each trace. C, A $4 \mathrm{kHz}$ tone. D, A $8 \mathrm{kHz}$ tone. $\boldsymbol{E}$, whole-cell modes to compare firing and synaptic response to the same binaural stimuli.

\section{Materials and Methods}

Animals. Thirty-seven GAD67-GFP knock-in mice (Ono et al., 2005; postnatal day 26-42 of either sex) were used for this study. A colony of knock-in mice backcrossed against a SwissWebster background was established. Breeding pairs consisted of one hemizygous and one wild type. Hemizygous offspring had high expression of GFP in their brains, and neonates (postnatal day $0-2$ ) were reliably phenotyped by checking for fluorescence under blue light. We used both the hemizygous littermates $(\mathrm{Tg} /+)$ and homozygous wild-type littermates $(+/+)$. Previous results showed no differences between $\mathrm{Tg} /+$ and $+/+$ mice in the peak time, peak amplitude, half-width, charge, or ratio of early and late charge of EPSCs or IPSCs (our unpublished observations). All experiments were done in accordance with institutional guidelines at the University of Connecticut Health Center and in accordance with National Institutes of Health Guidelines for the Care and Use of Laboratory Animals. All efforts were made to minimize the number of animals used and their suffering.

Sound stimulation. Acoustic stimuli were generated by a TDT System 2 or 3 (Tucker Davis Technologies) under the control of the customized MATLAB software (Brian Bishop, University of Connecticut Health Center, Farmington, CT; Marcel van der Heiden, University of Utrecht, Utrecht, The Netherlands). All sounds were delivered by electrostatic speakers (TDT EC1; Tucker Davis Technologies) through small metal tubes (Fig. $1 A, B$ ). The tip of the metal tube $(0.6 \mathrm{~mm}$; Fig. $1 B)$ was inserted in the external auditory meatus. The sound system was calibrated from 60 to 50,000 $\mathrm{Hz}$ for System 2 and from 100 to $100,000 \mathrm{~Hz}$ for System 3. The calibration was performed at the end of the metal tubes with a 1/4-inch microphone (type 4135; Brüel \& Kjær). In most experiments, we used tone bursts. In some experiments, we also used white noise. The sound duration was $200 \mathrm{~ms}$, and the rise and fall times were $5 \mathrm{~ms}$.

Surgical preparation. Animals were anesthetized with a mixture of ketamine $(100 \mathrm{mg} / \mathrm{kg})$, xylazine $(20 \mathrm{mg} / \mathrm{kg})$, and acepromazine $(10$ $\mathrm{mg} / \mathrm{kg}$ ) and maintained in an areflexive state with isoflurane mixed with oxygen during the surgery and recording. The breathing rate, heart rate, and blood oxygen saturation were monitored with a non-invasive vital sign monitor (MouseOx Plus; Starr Life Science), and the body temperature was monitored with a

The comparison of the responses to ipsilateral and contralateral stimuli. The power of the CM responses was plotted against the sound level. The gray lines indicate the binaural attenuation levels. Left, A $4 \mathrm{kHz}$ tone. The binaural attenuation level was $53.5 \mathrm{~dB}$. Right, A $8 \mathrm{kHz}$ tone. The binaural attenuation level was $71.6 \mathrm{~dB}$. $\boldsymbol{F}$, The ABLs at $2,4,6$, and $8 \mathrm{kHz}(n=3)$. 
Table 1. EPSCs and IPSCs in response to contralateral and ipsilateral stimuli

\begin{tabular}{llllr}
\hline Contra-EPSC & Contra-IPSC & Ipsi-EPSC & Ipsi-IPSC & $n$ \\
\hline+ & + & + & + & 18 \\
+ & + & - & - & 6 \\
- & - & + & + & 1 \\
+ & NA & + & NA & 6 \\
+ & NA & - & NA & 3 \\
NA & + & NA & + & 1 \\
\hline
\end{tabular}

Number of neurons with EPSCs and IPSCs to contralateral and ipsilateral stimuli. The symbols + and - indicate that the neuron had or did not have the response shown in the first line, respectively. NA indicates that the response was not tested.

rectal probe coupled to a digital thermometer and maintained at $>35^{\circ} \mathrm{C}$ with a chemical isothermal heating pad (Grabber). Surgery and recording were done in a double-walled sound attenuating chamber (IAC).

A small craniotomy was made over the right IC, and the dura was removed. After the craniotomy, the auditory brainstem response (ABR) to a click $(0.5 \mathrm{~ms})$ was measured to confirm a normal threshold for sound-evoked responses. After the ABR recordings, the head was fixed by a plastic screw glued on the scull, and the surface of the brain was covered with agarose gel ( $2 \%$ in $0.05 \mathrm{~m}$ phosphate buffer) to reduce the brain pulsation.

Cochlear microphonic recordings. To measure acoustic crosstalk, we measured the cochlear microphonic (CM) responses from one cochlea to ipsilateral and contralateral ear stimulation. The recording techniques have been detailed previously (Dallos, 1973). Briefly, the middle ear was opened, and a silver ball electrode was fixed in a position close to the round window of the cochlea. Tone pips were $20 \mathrm{~ms}$ duration $(5 \mathrm{~ms}$ rise/fall time) and presented every $50 \mathrm{~ms}$. Responses to 200 in-phase tones were averaged and then subtracted from the averaged response to 200 out-of-phase tones. The subtracted response was filtered by 1-octave-wide, four-pole bandpass filter centered at the tone pip frequency. The spectral analysis was performed using Clampfit 10.2 (Molecular Devices) with a segment length of 256 points and a rectangle-type window. The power of the CM response at the stimulus frequency was measured from the points between 4 and $16 \mathrm{~ms}$ after the tone onset. The baseline power level for each tone was calculated by measuring the power from the points in the last $12 \mathrm{~ms}$ of each $50 \mathrm{~ms}$ trace. The baseline power levels of 10 traces were averaged to calculate the mean and SD of baseline power levels. The threshold for the CM in the cochlea ipsilateral to the recording electrode was defined as the sound level that evoked a response that exceeded the baseline by 3 SDs. The binaural attenuation level is a measure of the acoustic crosstalk and was defined as the difference in the levels of the ipsilateral CM threshold and the corresponding contralateral CM response.

As expected, the CM to ipsilateral ear stimulation was always larger compared with that after contralateral ear stimulation. Figure 1 shows the CMs to ipsilateral and contralateral ear stimulation for a $4 \mathrm{kHz}$ tone (Fig. 1C) and an $8 \mathrm{kHz}$ tone (Fig. 1D) at different sound levels. At $4 \mathrm{kHz}$, a $\mathrm{CM}$ response was only visible when the contralateral ear level was 110 dB SPL (Fig. 1C) and is plotted in Figure 1C. At $8 \mathrm{kHz}$, the CM to contralateral ear stimulation did not exceed the ipsilateral threshold (Fig. $1 D)$. In this case, we measured the difference between the maximum ipsilateral level and the point of the maximum contralateral response (Fig. $1 E$ ). Figure $1 F$ plots the binaural attenuation level as a function of frequency and indicates that acoustic crosstalk systematically decreases with stimulation frequency.

Whole-cell recordings. Micropipettes were made from borosilicate glass capillaries (34502-99; Kimble Chase) pulled on a Flaming/Brown P80/PC (Sutter Instruments) or PC-10 (Narishige) electrode puller. They were filled with a Cs-gluconate-based internal solution (125 mM Csgluconate, $5 \mathrm{~mm} \mathrm{CsCl}, 5 \mathrm{~mm}$ tetraethylammonium-Cl, $10 \mathrm{~mm}$ HEPES$\mathrm{KOH}, 1 \mathrm{~mm}$ EGTA, $4 \mathrm{~mm}$ MgATP, $0.3 \mathrm{~mm} \mathrm{Na} \mathrm{Na}_{2} \mathrm{GP}, 10 \mathrm{~mm}$ phosphocreatine, 1.5-6 mM QX-314, 0.2\% Neurobiotin, $\mathrm{pH} 7.3$ ), and their resistance was 3-5 M $\Omega$. Whole-cell recordings were achieved using the standard blind technique (Margrie et al., 2002). For voltage-clamp recording, the pipette $(5-20 \mathrm{pF})$ capacitance was measured and cancelled before a break-in. The membrane capacitance and series resistance were compensated after a break-in. The series resistance $(54.9 \pm 2.7 \mathrm{M} \Omega, n=$ 40) was compensated for $60-70 \%$. The liquid junction potential was estimated to be $10 \mathrm{mV}$ and corrected (Sun et al., 2010). The data were filtered at $4 \mathrm{kHz}$ and sampled at $10 \mathrm{kHz}$ using Multiclamp 700B Amplifier, Digidata 1440A digitizer, and Clampex 10.2 system (Molecular Devices).

Separation of EPSC and IPSC. To separate the excitatory and inhibitory synaptic inputs, neurons were clamped at the assumed reversal potentials of EPSC and IPSC, and the responses to sound were recorded. Because EPSCs in IC are suggested to be glutamatergic and IPSCs are GABAergic or glycinergic (Caspary et al., 1990), we assumed reversal potentials of EPSC $\left(E_{\text {EPSC }}\right)$ and IPSC $\left(E_{\text {IPSC }}\right)$ as 0 and $-65 \mathrm{mV}$, respectively. For estimating the $E_{\mathrm{IPSC}}$, we used a $\mathrm{Cl}^{-}$concentration of $115 \mathrm{~mm}$ based on its concentration in mice blood (104-120 mm; Charles River Laboratories). These reversal potentials were confirmed in our own laboratory in experiments to block ionotropic glutamate, GABA, and glycine receptors in vivo (data not shown).

Recording procedure. Neurons were first clamped at $-65 \mathrm{mV}$ to record the EPSC. For 35 neurons, we presented tone bursts of $50-70 \mathrm{~dB}$ to determine the best frequency (BF) of the neuron. BF was determined as the frequency that evoked the largest EPSC. The recorded BFs were 2-22 $\mathrm{kHz}$ (mean $\pm \mathrm{SEM}, 12.8 \pm 0.73 \mathrm{kHz}, n=35)$. For six neurons, we used a band-limited noise from 10 to $50 \mathrm{kHz}$.

First, we determined the threshold as the sound level that evoked a detectable synaptic response. We presented sounds to the ear contralateral to the recording electrode, changing the sound level randomly from 0 to $80 \mathrm{~dB}$ SPL in $10 \mathrm{~dB}$ steps. Second, the same stimulus protocol was delivered to the ipsilateral ear. Finally, we simultaneously stimulated the contralateral and ipsilateral ears, namely, binaural stimulation. We used two different binaural stimulation methods (Irvine, 1987). For the constant ABL method, the contralateral and ipsilateral intensities were varied symmetrically in 5 or $10 \mathrm{~dB}$ steps about a specified ABL. In the contralateral-level (CL)-constant method, the contralateral sound level was held constant (at 10-30 dB above the threshold), and the ipsilateral sound level was varied between 0 and $80 \mathrm{~dB}$ SPL in $10 \mathrm{~dB}$ steps. In both methods, the $200 \mathrm{~ms}$ sounds were presented every 700 or $1200 \mathrm{~ms}$ and repeated 10-20 times. When there was visible synaptic depression, the repetition interval was increased to $2200 \mathrm{~ms}$. After we recorded the responses at $-65 \mathrm{mV}$, we changed the holding potential to $0 \mathrm{mV}$ and repeated the same sound protocol as above.

In five neurons, we compared the time course of the evoked firing pattern and the evoked currents. We first recorded the evoked spikes extracellularly in a giga-seal configuration before a break-in. The extracellular spikes were recorded in voltage-clamp mode instead of the conventional current-clamp mode to improve the stability during the transition from giga-seal extracellular and whole-cell intracellular recording. We validated that there is no difference in the recorded spike activity between voltage clamp and current clamp (data not shown). After recording, the spike traces were high-pass filtered at $300 \mathrm{~Hz}$ (see Figs. 9, 10).

Synaptic current analyses. Most analyses were performed using Clampfit 10.2 software (Molecular Devices) and Origin 7.5 (OriginLab). The spontaneous EPSCs and IPSCs were collected from the recordings of 5-10 s duration. Sound-evoked EPSCs and IPSCs were averaged. If noise levels were too high, some traces were excluded, and, if fewer than eight traces were available for averaging, the entire recording was removed from the dataset. After averaging, EPSCs and IPSCs were subtracted from the baseline and divided by the driving force to convert them into conductance. To evaluate the size of the synaptic inputs, we used two parameters: (1) the peak amplitude; and (2) the charge transfer of the currents. The peak amplitude was measured from the baseline. The charge transfer was measured as the evoked currents integrated through time. The onset latency of synaptic input was identified as the time at which the current amplitude exceeded 2 SDs of baseline fluctuation. Data are given as mean \pm SEM. The series resistance voltage errors were estimated from the uncompensated series resistance and holding currents. We excluded data with a series resistance voltage error of $>10 \mathrm{mV}$. Data were subjected to statistical evaluation using the $F$ test, the Student's $t$ test, or a 
one-way ANOVA with post hoc comparison. Criteria for significance were defined as $p<$ 0.05 .

All of these results take into consideration the difficulty of measuring synaptic current in vivo in which uncompensated series resistance may lead to errors in measurement especially of current amplitude. For this reason, these results are presented as relative, within cell measures or normalized comparisons of monaural and binaural responses.

Chemicals. Neurobiotin was from Vector Laboratories. All others were from Sigma-Aldrich.

\section{Results}

The present findings were based on voltage-clamp recordings of 40 IC neurons in the mouse. The depth of the recording sites (207-998 $\mu \mathrm{m}$, mean of $415.1 \pm 27.8 \mu \mathrm{m}, n=40)$ and the latency of the onset of the synaptic current indicate that most neurons were located in the central nucleus of the IC. The latency evoked by contralateral or ipsilateral stimuli was uniformly short and not significantly different among the four groups (contra-EPSC, ipsi-EPSC, contra-IPSC, and ipsi-IPSC; ANOVA, $p=0.58$ ). The median onset latency of each group was $\sim 15$ ms, with most neurons between 10 and $20 \mathrm{~ms}$ (range of $8.9-36.3 \mathrm{~ms}$, except one that could be an off response). These latencies are consistent with our extracellular recordings in the central nucleus of the IC of these same mice with identical anesthetic and stimulus conditions.

One important question is to what extent the binaural response is predicted by the monaural responses. To relate the responses to the binaural ABL stimuli with those to monaural stimuli, we recorded both in most neurons. However, we will first present the responses to monaural and binaural stimuli separately and then examine their relationship. Next, we ask how responses to ABL stimuli compare with the more common CL-constant method of assessing ILD processing. Finally, we make a direct comparison of synaptic currents and firing pattern to ABL stimuli in neurons in which both were recorded.

\section{Monaural: both EPSCs and IPSCs were evoked by sounds in either ear}

For 35 of the 40 neurons, the monaural responses of an IC neuron to BF tones always included both an EPSC and an IPSC (Table 1). For example, Figure $2 A$ shows the responses of a neuron to $17 \mathrm{kHz}$ tones presented to each ear alone at different sound levels. For contralateral and ipsilateral stimulation, both EPSCs (red traces) and IPSCs (blue traces) were evoked with stimuli at $20 \mathrm{~dB}$ SPL (Fig. 2A). As the tone level increased, the currents became larger. When both EPSCs and

A
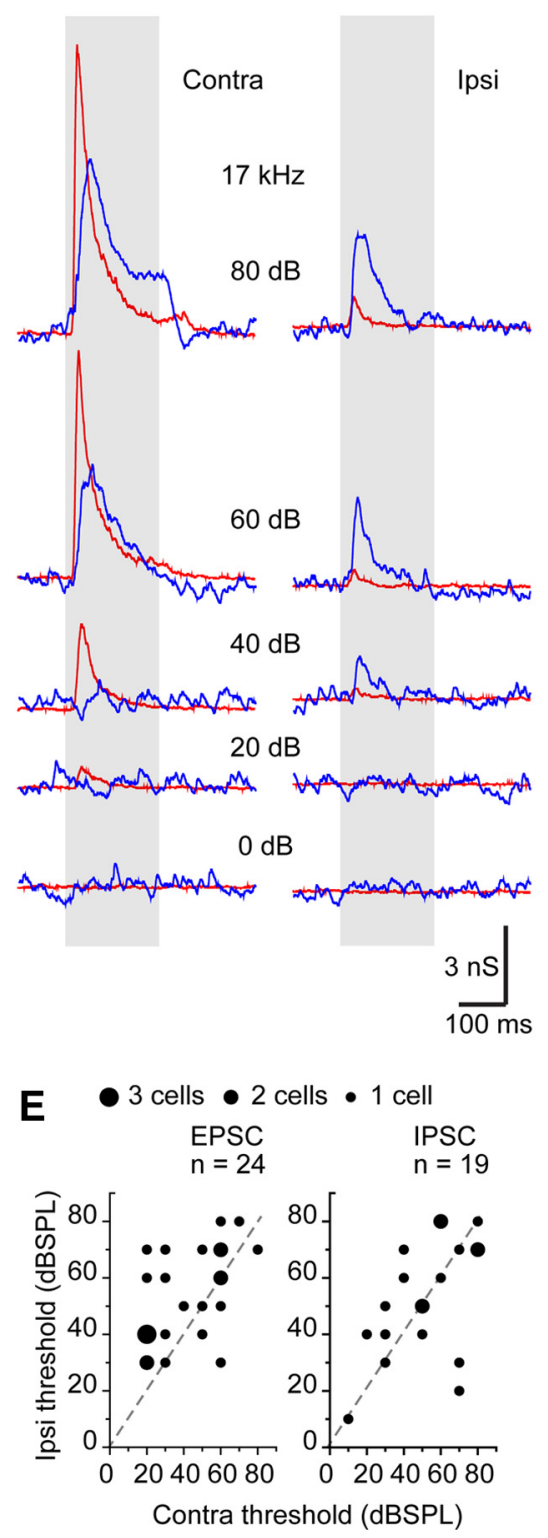

Figure 2. The most IC neurons had excitatory and inhibitory inputs to monaural contralateral and ipsilateral sound stimuli. $\boldsymbol{A}$, The representative synaptic responses of $\mathrm{IC}$ neurons to monaural contralateral and ipsilateral BF tone stimuli. The tone intensities and the BF are shown. The red traces are $10 \times$ averaged EPSCs, and the blue traces were $10 \times$ averaged IPSCs. The evoked current responses were converted into the conductance and presented to accelerate the comparison between EPSC and IPSC. B, C, In most neurons, the contralateral EPSCS $(\boldsymbol{B})$ and IPSCS (C) were larger than the ipsilateral EPSCS $(\boldsymbol{B})$ and IPSCS (C). The ratios of the peak and charge of the ipsilateral responses to those of contralateral responses were plotted against the sound level. Dotted lines indicate that the ratio was equal to 1 . The ratio $<1$ indicates that the contralateral response is larger than the ipsilateral response. $D$, The number of the cells with three types of evoked charge sound-level functions. M, Monotonic; S, saturated; N, nonmonotonic. $\boldsymbol{E}$, The comparison of the thresholds of contralateral and ipsilateral responses. The different dot sizes indicate the different number of cells. Dashed lines are unity lines. $\boldsymbol{F}$, The comparison of the thresholds of EPSCs and IPSCs. contra-EPSCs, $44.0 \pm 4.0 \mathrm{~dB}, n=24$; ipsi-EPSCS, $54.0 \pm 3.3 \mathrm{~dB}, n=24$; contra-IPSCs, $51.6 \pm 4.9 \mathrm{~dB}, n=19$; ipsi-IPSCs, $52.6 \pm 4.8 \mathrm{~dB}, n=19$.
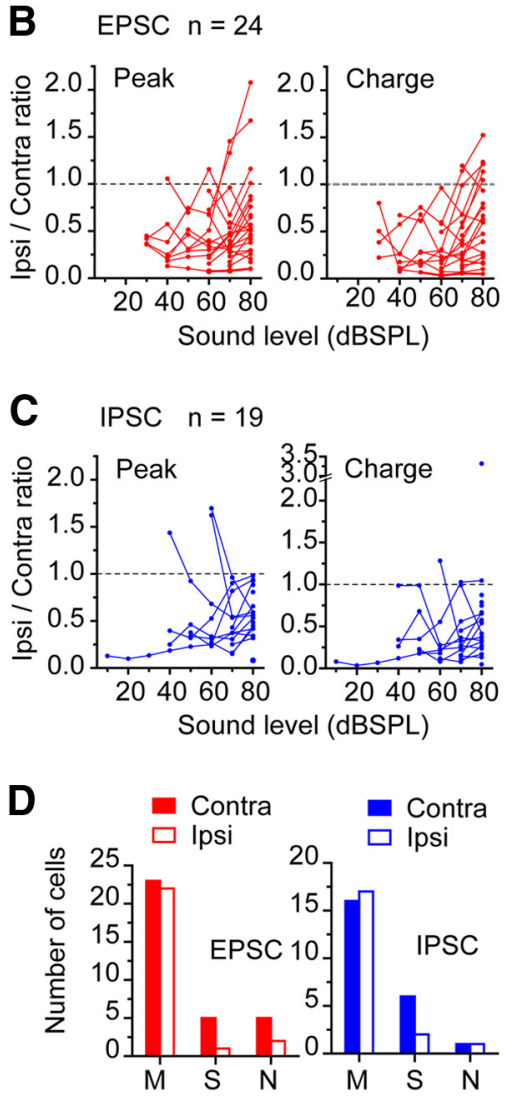

\section{F}

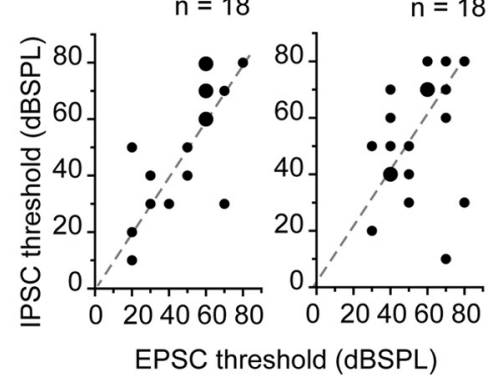



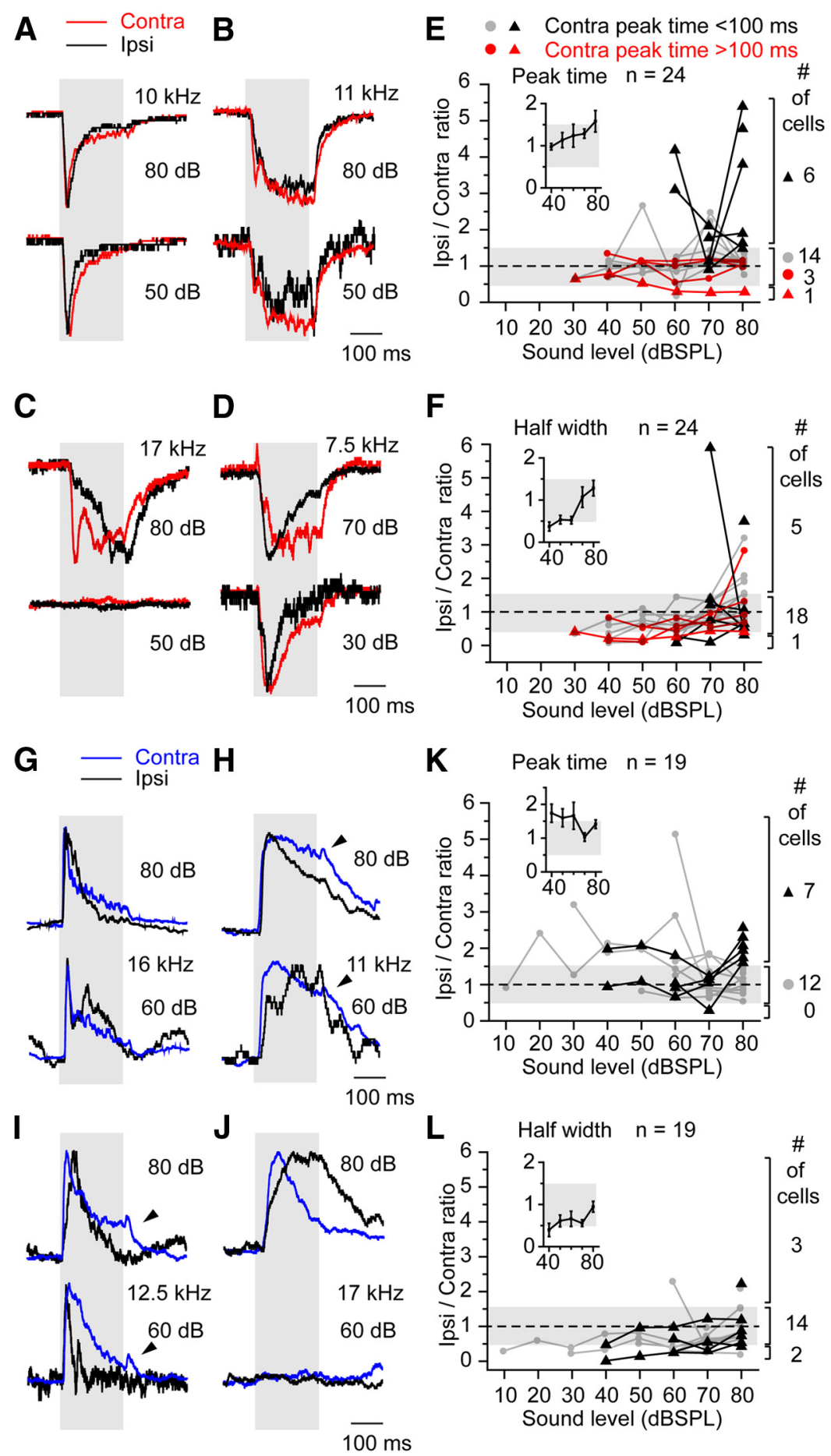

Figure 3. The comparison of the time courses of the contralateral and ipsilateral responses. $\boldsymbol{A}-\boldsymbol{D}$, The representative traces of the EPSCS. The contralateral responses are red, and the ipsilateral responses are black. The amplitudes of the traces are normalized. $\boldsymbol{A}, \boldsymbol{B}$, Contralateral-ipsilateral matched responses ( $\boldsymbol{A}$, transient; $\boldsymbol{B}$, buildup). $\boldsymbol{C}, \boldsymbol{D}$, Contralateral-ipsilateral unmatched responses. $\boldsymbol{E}, \boldsymbol{F}$, The ratio of the peak time $(\boldsymbol{E})$ and half-width $(\boldsymbol{F})$ of contralateral and ipsilateral responses were plotted against the sound level. Gray bars indicate the ratio from 0.5 to 1.5 . The data were grouped into four types by the peak time of the contralateral response and ipsilateral/contralateral peak time ratio at $80 \mathrm{~dB}$. At the right, the numbers of the each type of neuron $(\boldsymbol{E})$ or the numbers of the cells with a ratio of $<0.5,0.5-1.5$, or $>1.5(\boldsymbol{F})$ are shown. At $80 \mathrm{~dB}$, the peak times of the contra-EPSCs ranged from 15.5 to 403.7 ms $(65.5 \pm 14.1 \mathrm{~ms}, n=34)$, whereas those of the ipsi-EPSCs ranged from 17.8 to $447.9 \mathrm{~ms}(93.2 \pm 21.3 \mathrm{~ms}, n=24)$. The half-widths of the contra-EPSCs ranged from 2.6 to $186.1 \mathrm{~ms}(44.6 \pm 6.7 \mathrm{~ms}, n=34$ ), whereas those of the ipsi-EPSCs ranged from 10.6 to $170.6 \mathrm{~ms}(54.1 \pm 8.9 \mathrm{~ms}, n=24) . \mathbf{G}-\boldsymbol{J}$, The representative traces of IPSCs. The contralateral responses are blue, and the ipsilateral responses are black. $\boldsymbol{G}$, Contralateral-ipsilateral matched responses. $\boldsymbol{H}$, I, Slower ipsilateral response. J, Slower contralateral response. $\boldsymbol{K}, \boldsymbol{L}$, The ratio of the peak time $(\boldsymbol{K})$ and half-width $(\boldsymbol{L})$ of contralateral and ipsilateral responses were plotted against the sound level. The data were grouped into two types by the peak time of the contralateral response and ipsilateral/ contralateral peak time ratio at $80 \mathrm{~dB}$. At $80 \mathrm{~dB}$, the peak times of the contra-IPSCs ranged from 19.9 to $86.0 \mathrm{~ms}$
BF of most neurons was higher than 7.5 $\mathrm{kHz}(12.1 \pm 0.68 \mathrm{kHz}, n=25)$ in which acoustic attenuation between the ears is $>60 \mathrm{~dB}$ in our closed sound delivery system (Fig. $1 F)$.

The EPSCs and IPSCs to ipsilateral sounds were usually smaller than those evoked by contralateral sounds (Fig. $2 A$ ). The ratio of the responses is $<1$ for both EPSC and IPSC peak amplitude and charge over the range of sound levels tested. The mean ipsilateral/contralateral ratio of the EPSC peak amplitudes at 80 $\mathrm{dB}$ was $0.64 \pm 0.10$ and charge $0.63 \pm 0.10$ $(n=24)$, whereas the mean ipsilateral/ contralateral ratio of the IPSC peak amplitudes was $0.55 \pm 0.05$ and charge $0.59 \pm$ $0.16(n=19)$.

Three types of charge-level functions relative to increasing sound levels were seen: (1) monotonic; (2) saturating; and (3) nonmonotonic (Fig. 2D, labels M, S, $\mathrm{N}$, respectively). The charge-level function is similar to a rate-level function except that current charge is used instead of spike rate. Responses were classified as monotonic if the charge-level function increased systematically with level, as saturating if it increased and then plateaued with level ( $<20 \%$ from maximum), and as nonmonotonic if it increased and then decreased with level (decrease $>20 \%$ from maximum). The dominant type was monotonic in both contralateral and ipsilateral EPSCs and IPSCs (Fig. 2D). There was no significant difference in the proportion of each type among EPSCs and IPSCs from contralateral and ipsilateral ears $\left(p=0.114, \chi^{2}\right.$ test $)$.

Comparisons of thresholds for contralateral or ipsilaterally evoked currents (Fig. 2E,F) showed that thresholds of EPSCs from ipsilateral stimuli were higher than those of EPSCs from contralateral stimuli (Fig. $2 E ; p=0.0459$, paired $t$ test, Ryan's method, one-way ANOVA, $p=$ $0.288)$. However, there was no significant difference between the other pairings.

Monaural: matched temporal responses to contralateral and ipsilateral stimuli In most neurons, the EPSCs showed a similar time course to both contralateral and ipsilateral stimuli (Fig. 3A,B). The onset of the EPSCs to contralateral stimuli

$\longleftarrow$

$(46.3 \pm 3.5 \mathrm{~ms}, n=24)$, whereas those of the ipsi-IPSC ranged from 17.6 to $180.4 \mathrm{~ms}(63.5 \pm 10.1 \mathrm{~ms}, n=19)$. The half-width of the contra-IPSCs ranged from 18.1 to $262.4 \mathrm{~ms}$ $(80.5 \pm 13.3 \mathrm{~ms}, n=24)$, whereas those of the ipsi-IPSCs ranged from 6.1 to $226.7 \mathrm{~ms}(74.9 \pm 16.9 \mathrm{~ms}, n=19)$. 
was usually fast rising (Fig. $3 A$ ) or, less often, a buildup response (Fig. 3B). The peak time of the ipsilateral EPSC usually matched that of the contralateral EPSC. The neurons with unmatched EPSCs usually were faster in response to the contralateral stimuli (Fig. 3C) with one exception (Fig. 3D).

Although the peak times of the EPSCs were similar, the difference between the contralateral and ipsilateral times became larger as the sound level increased (Fig. 3E, inset). This difference was largest at the highest level, $80 \mathrm{~dB}$, at which the ipsilateral/contralateral ratio was $1.58 \pm 0.25$. The ipsilateral EPSCs were slower than the contralateral EPSCs in their peak times. Thus, we compared the time courses of the contralateral and ipsilateral responses at $80 \mathrm{~dB}$.

Most neurons $(71 \%, 17$ of 24 at $80 \mathrm{~dB})$ had EPSCs that were well matched in peak time within the 0.5-1.5 ratio range (Fig. $3 E$, gray box). Fourteen neurons had fast rising EPSCs (gray circle), and three had buildup EPSCs (red circle). Only onequarter of neurons (Fig. 3C) had much slower responses to ipsilateral stimuli (ratio $>1.5$; Fig. $3 E$, black filled triangle), and the threshold of their ipsilateral EPSCs was higher, unlike the well matched neurons $(>1.5,66.7 \pm 3.3 \mathrm{~dB}, n=6 ;<1.5$, $50.0 \pm 3.8 \mathrm{~dB}, n=18$; $p=0.0038$, Student's $t$ test). Only one neuron showed a faster ipsilateral response (Fig. 3D, E, red filled triangle). Also well matched were the durations of the responses measured as $50 \%$ half-width of the EPSC ( 18 of 24 ; Fig. $3 F$, gray box). In general, the higher threshold ipsilateral EPSCs had shorter durations at lower level but longer durations at higher level (Fig. 3F, inset).

For IPSCs, the time courses of the monaural responses were even more similar than that seen in the EPSCs, and the time course was less influenced by stimulation level. The ipsilateral/contralateral peak times of IPSCs were well matched (Fig. $3 G, H, K ; n=12$ ) or showed slower responses to ipsilateral stimuli (Fig. $3 I, J, K ; n=7)$. Similarly, the half-width of IPSCs also was well matched in most neurons (Fig. $3 L ; n=14$ ). In summary, most synaptic responses to monaural stimuli in the contralateral and ipsilateral ears were well matched in their time course.

\section{Binaural: balanced EPSCs and IPSCs to}

\section{ABL-constant stimuli}

We used the ABL method to simulate a sound source at different azimuthal locations (Irvine, 1987). The ILD was changed, but the ABL was constant (Fig. 4A). The ILD is the primary binaural cue for sound localization in mice, a high-frequency biased animal (Allen and Ison, 2010; Lauer et al., 2011).

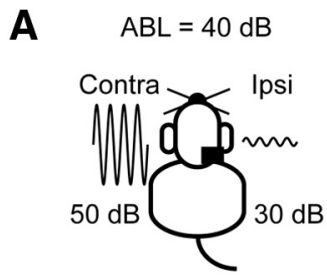

$I L D=-20 d B$

B

Contra-preferred
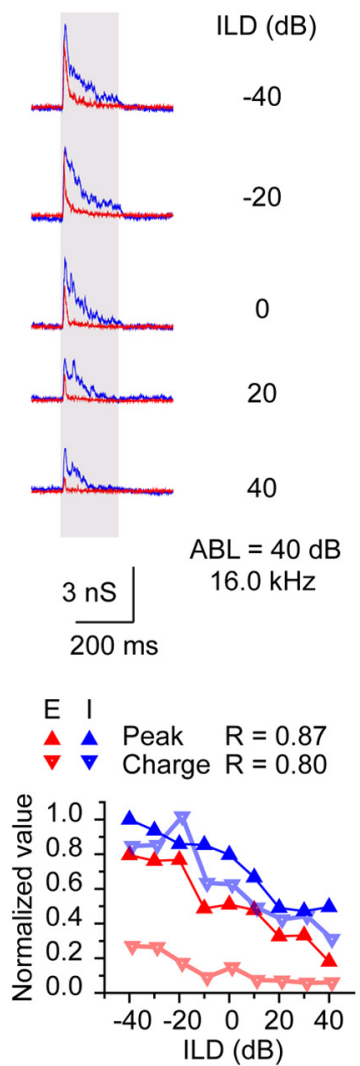

E

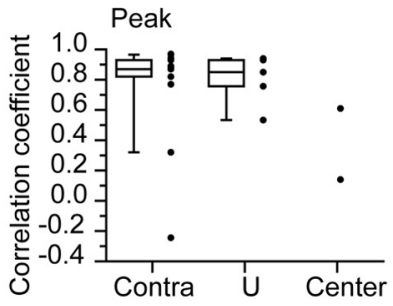

Recording site

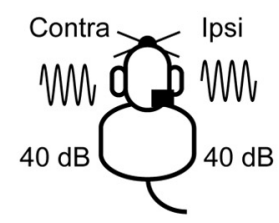

$\mathrm{ILD}=0 \mathrm{~dB}$

C

U-shape

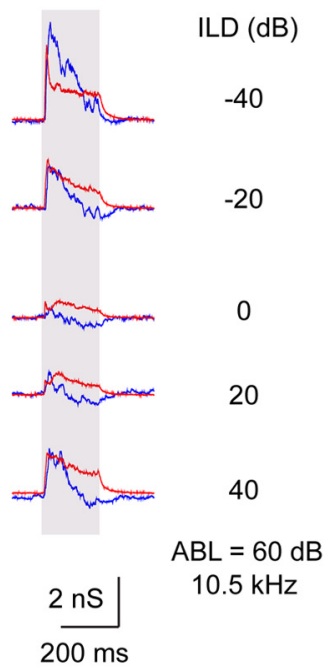

Peak $R=0.93$
Charge $R=0.80$

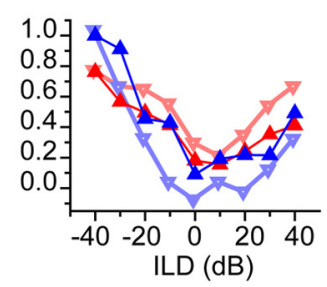

D

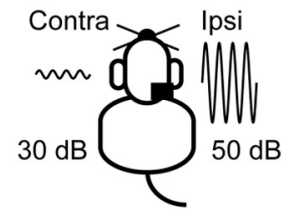

ILD $=20 \mathrm{~dB}$

\section{Center-preferred}

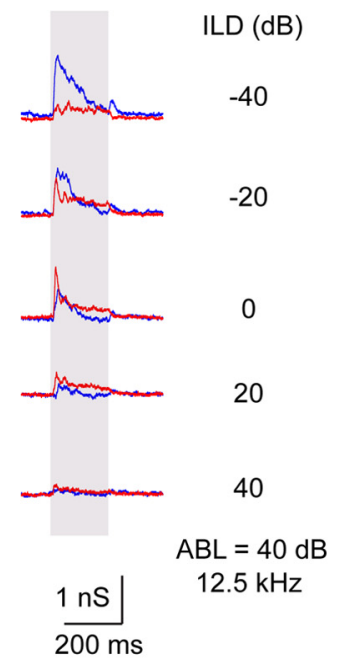

Peak $\quad R=0.14$

Charge $\mathrm{R}=0.52$

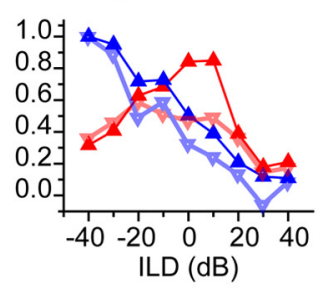

Figure 4. Synaptic responses of $I C$ neurons to $A B L$ stimuli. $A$, Schematic drawings of ABL stimuli. ABL was made $40 \mathrm{~dB}$ SPL, and the ILD was changed. $B$, Contra-preferred neuron. $C$, $U$-shaped neuron. $\boldsymbol{D}$, Center-preferred neuron. BF of each neuron was shown. Top, The traces of the synaptic responses. Bottom, The normalized plots of the peak amplitude and the charge of the responses against ILD. The correlation of coefficients between the binaural responses of EPSC and IPSC are shown. $E$, Population data of correlation coefficients between the binaural responses of EPSCs and IPSCs. For the contra-preferred neuron $(n=13)$ and the U-shaped neuron $(n=6)$, box plots are presented with raw data points. For center-preferred neurons $(n=2)$, only raw data points were presented. The whiskers represent 10 and 90 percentiles.
The EPSCs had three patterns in response to ABL stimuli in 30 neurons studied (Fig. $4 B-D$ ): the contra-preferred pattern, the U-shaped pattern, and the center-preferred pattern. The neurons with contra-preferred responses showed larger EPSCs when the contralateral level is higher (negative ILD) than the ipsilateral 
A Contra-preferred
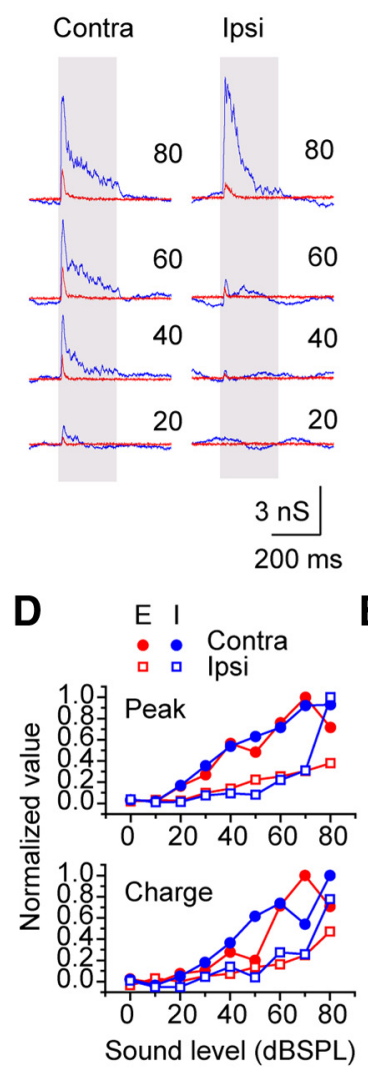

B U-shape

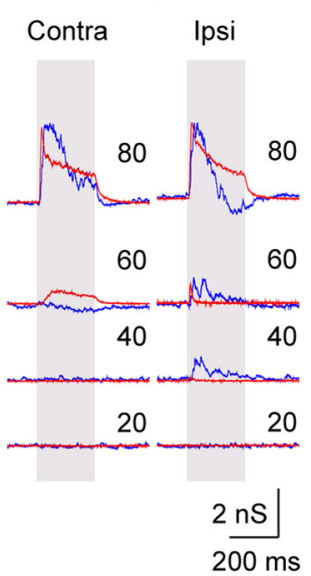

E
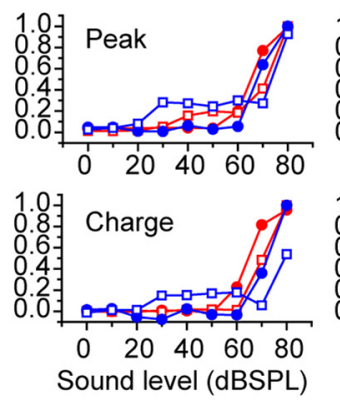

C

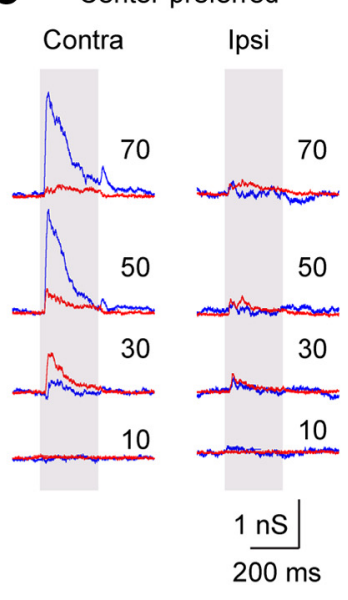

$\mathbf{F}$
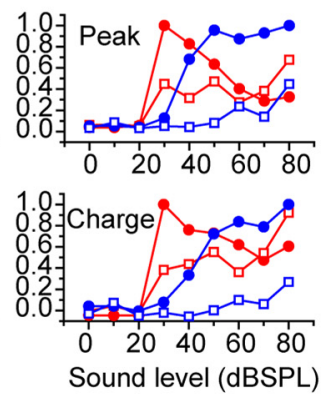

G

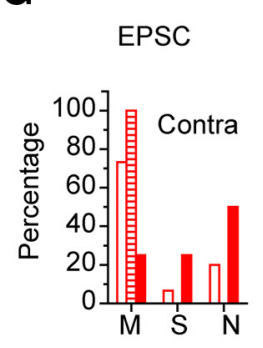

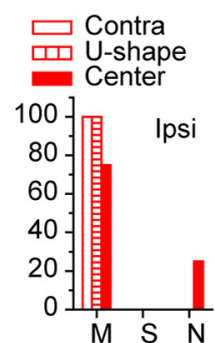

H

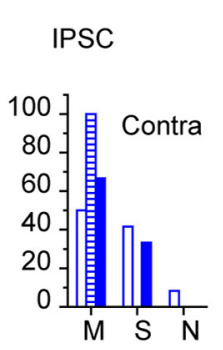

Figure 5. Monaural responses of the neuron of each $A B L$ response type. $\boldsymbol{A}$, Contra-preferred neuron. $\boldsymbol{B}, \mathrm{U}$-shaped neuron. $\boldsymbol{C}$, Center-preferred neuron. $\boldsymbol{A}-\boldsymbol{C}$ are the recordings from the same neurons as $\boldsymbol{B}-\boldsymbol{E}$. $\boldsymbol{D}-\boldsymbol{F}$, The normalized plots of the peak amplitude (top) and charge (bottom) sound-level functions in these neurons. $\boldsymbol{D}-\boldsymbol{F}$ correspond to $\boldsymbol{A}-\boldsymbol{C}$, respectively. $\boldsymbol{G}, \boldsymbol{H}$, The percentage of the cells with three types of evoked charge sound-level functions in each binaural response type to ABL stimuli. M, Monotonic; $S$, saturated; N, nonmonotonic. $\boldsymbol{G}$, EPSCS. $\boldsymbol{H}$, IPSCS.

level and smaller EPSCs when the ipsilateral level is higher (positive ILD; Fig. 4B). The contra-preferred response was the most prevalent $(n=19$ of 30$)$. Neurons with the U-shaped response ( $n=6$ of 30) showed large EPSCs at the negative and positive ILDs with a small response near 0 ILD (Fig. 5C). In contrast, the neurons with the center-preferred response $(n=5$ of 30$)$ showed the largest EPSCs near 0 ILD (Fig. 4D). In four neurons with no responses to ipsilateral stimuli, we examined the EPSCs and IPSCs to ABL stimuli, and these all showed the contra-preferred responses.

The IPSCs evoked by the ABL stimuli were usually well correlated to the EPSCs. In neurons with the contra-preferred and U-shaped responses, the size of the IPSC and EPSC were well correlated over the range of ILDs (Fig. $4 B, C, E$ ). In contrast, neurons with the center-preferred responses had poorly correlated EPSCs and IPSCs (Fig. 4D,E). Interestingly, the IPSC of the

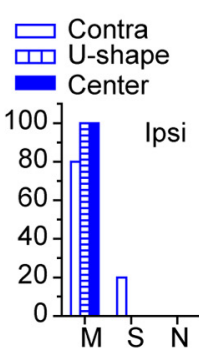

center-preferred neurons had a contrapreferred pattern, despite the preference of the EPSC for the center. In summary, for most IC neurons, the EPSCs and IPSCs follow the changing ILDs in a well correlated, balanced manner, that is, the IPSCs follow the changes of ILD in proportion to EPSCs.

Binaural: role of monaural inputs in shaping the responses to ABL stimuli Monaural response properties distinguished neurons with center-preferred responses to ABL stimuli from other neurons. The neurons with contra-preferred responses had all types of charge-level functions (Fig. $5 A, D, G$ ), but the monotonic response was dominant (Fig. 5G; the neuron in Fig. $5 A$ is also shown in Fig. $2 A$ ). In contrast, all neurons with U-shaped responses had EPSCs and IPSCs, showing only a monotonic charge-level function (Fig. $5 B, E, G$ ). The EPSCs of the neurons with center-preferred responses also differed because they showed more nonmonotonic and saturating charge-level functions (Fig. 5C, $F, G$ ), whereas their charge-level functions for IPSCs were similar to other types (Fig. $5 H$ ).

We asked whether the sum of the monaural EPSCs could predict the responses to the binaural $\mathrm{ABL}$ stimuli. The monaural EPSCs were summed to create a predicted binaural response for each neuron (Fig. 6, red). The mean predicted response for the group is shown in black (Fig. 6). In the neurons with contra-preferred and $\mathrm{U}$-shaped responses, the mean predicted responses were similar to the binaural patterns (Fig. 6A,B). However, in the neurons with center-preferred responses, the mean predicted response lacked a maximal response at the center and was less similar to the binaural pattern (Fig. 6C).

Whether monaural EPSCs can predict binaural responses may be an indication of the extent binaural inputs from lower centers contribute to the response to ILD. To test the accuracy of monaural EPSCs predicting the binaural EPSC, we modeled the predicted EPSC for each neuron by summing the two monaural EPSCs (Fig. $7 A-D$, black). We then subtracted predicted response from the actual binaural EPSC obtained with the ABL stimulus (ABL; Fig. 7A-D, red, ABL) to obtain a difference curve (Fig. 7A-D, green, Sub). We limited this analysis of EPSCs and IPSCs (below) to neurons in which the monaural and binaural responses were both recorded with the same stimulus repetition rate ( $n=11$ neurons; see Materials and Methods).

For neurons with the contra-preferred responses, the difference between the actual and the predicted response to $\mathrm{ABL}$ stimuli was larger for ipsilateral stimuli than that for contralateral stimuli (Fig. $7 A, B$ ). Six of seven neurons with contra-preferred responses had binaural responses that were smaller than the predicted response $(<1.0$; Fig. $7 G$, left), and 
the mean showed that the difference became larger as the ILD moved from negative to positive (Fig. $7 G$, left). One neuron showed a larger binaural response than predicted response. For neurons with U-shaped responses, the binaural and predicted responses were well matched, indicating little if any binaural input in two of three neurons. However, in a third neuron, the binaural response was four times larger than predicted positive ILDs, indicating a strong binaural input driven by the ipsilateral ear (Fig. 7G, right). For the one neuron with center-preferred response available for this analysis, there was a clear binaural effect at ILDs near $0 \mathrm{~dB}$. This shaped the center-preferred binaural response and differed sharply from the predicted curve that resembled the contra-preferred pattern (Fig. 7D, G, right).

Despite the small number of neurons available for modeling, the data show that, for most neurons, the summation of inputs during binaural ABL stimulation produces EPSCs that can differ in size from those predicted from the monaural responses. In most cases, the binaural EPSC is smaller than predicted but in a few cases is much larger. This indicates a binaural interaction in a center below the IC that either decreases or increases the excitatory input to the IC during binaural stimulation. This size difference is not attributable to differences in data collection methods because we limited the number of cells in this analysis to those in which the same repetition rate was used for monaural and binaural stimulation. Thus, this difference is not attributable to differential adaptation.

The IPSCs showed less differences between the binaural and predicted responses than the EPSCs $(n=9$; Fig. $8 E, H, I)$. In only two neurons was the binaural IPSC less than the prediction from monaural responses (Fig. $7 F$ ). The mean of the binaural/prediction ratios was close to one and flat across all ILD levels, and increased binaural IPSCs relative to predicted were not seen. This suggests that, in the inhibitory pathways, there was less binaural interaction at lower centers than in the excitatory pathways.

\section{Binaural: effects of varied ipsilateral levels during fixed contralateral levels}

In addition to the ABL method, we used the CL-constant protocol (see Materials and Methods) to generate a second type of acoustic stimulus to study the effects of ILD in 27 neurons. Four types of EPSC response patterns were seen with the CLconstant stimuli in which the level of contralateral stimulus was held constant and the level of ipsilateral stimulus was varied. The patterns were suppression (Fig. $8 A$ ), enhancement
B

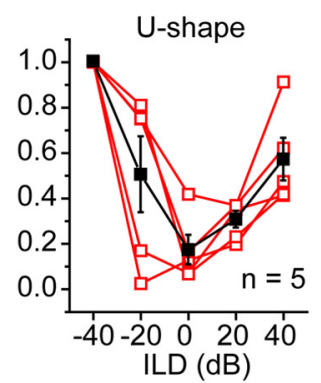

C
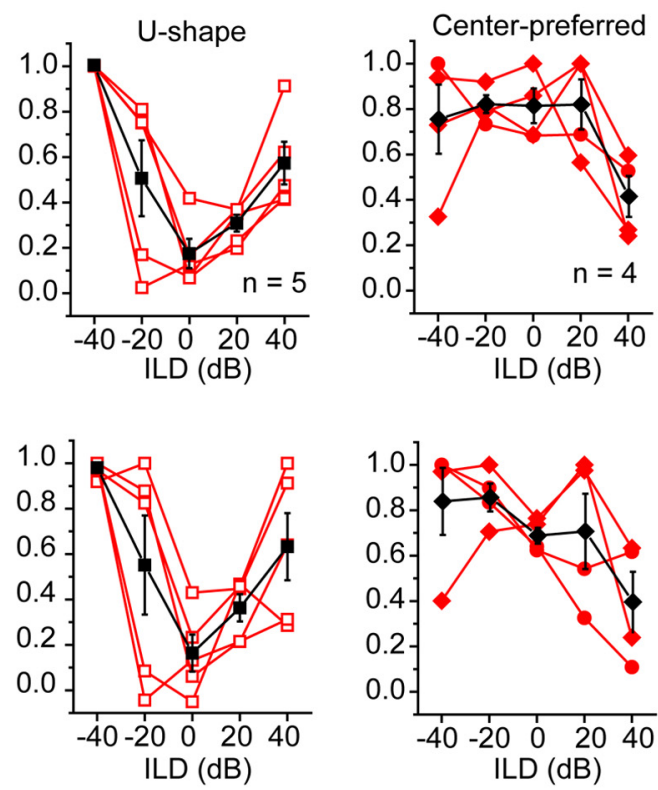

E

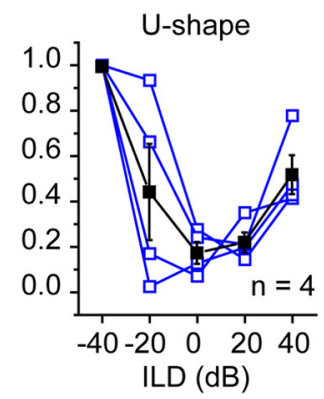

$\mathbf{F}$
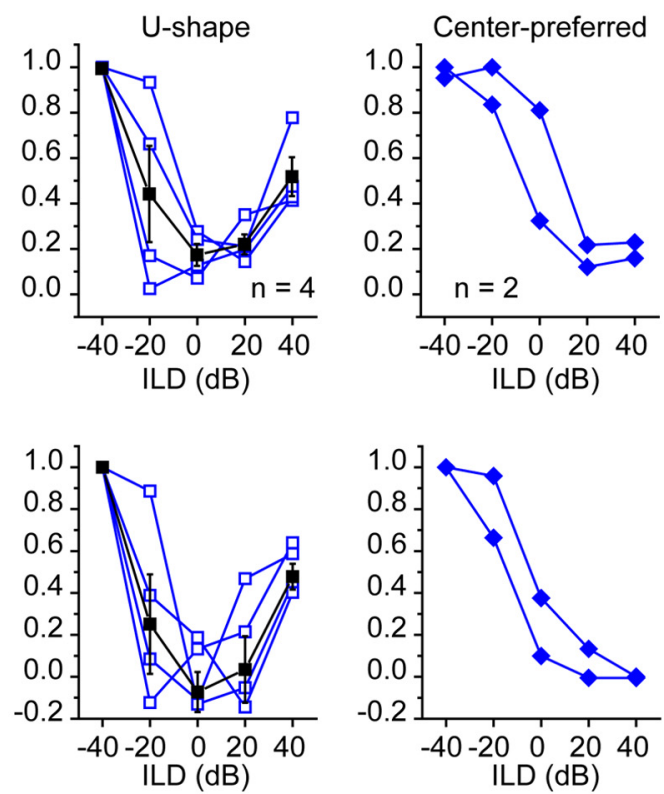

Figure 6. The binaural response curves predicted from monaural responses. $\boldsymbol{A}-\boldsymbol{C}$, EPSCS. D-F, IPSCs. A, D, Contra-preferred. $\boldsymbol{B}$ $\boldsymbol{E}, \boldsymbol{U}$-shapes. $\boldsymbol{C}, \boldsymbol{F}$, Center-preferred. The predicted curves were drawn by adding the contralateral and ipsilateral responses. Red lines are the predicted curves from the different neurons. Black lines are averaged curves.

(Fig. 8B), nonmonotonic enhancement (Fig. 8C), and no change (Fig. $8 D$ ). In the suppression pattern, the peak amplitude and charge of the EPSC decreased as the level increased in the ipsilateral ear (Fig. 8A). In contrast, with the enhancement pattern, the EPSC increased with increasing ipsilateral sound level. In the case of nonmonotonic enhancement, the EPSC saturated before reaching the maximum level of $80 \mathrm{~dB}$ (Fig. $8 C, \sim 30 \mathrm{~dB}$ ). The fourth type showed little change in the EPSC in response to changing ipsilateral level (Fig. 8D).

We also recorded the IPSCs to CL-constant stimuli in 13 neurons. There were three types of response patterns: (1) suppression (Fig. 8E); (2) enhancement (Fig. 8F); and (3) no change (Fig. 8G). Nonmonotonic enhancement of the IPSCs was not seen. 
A

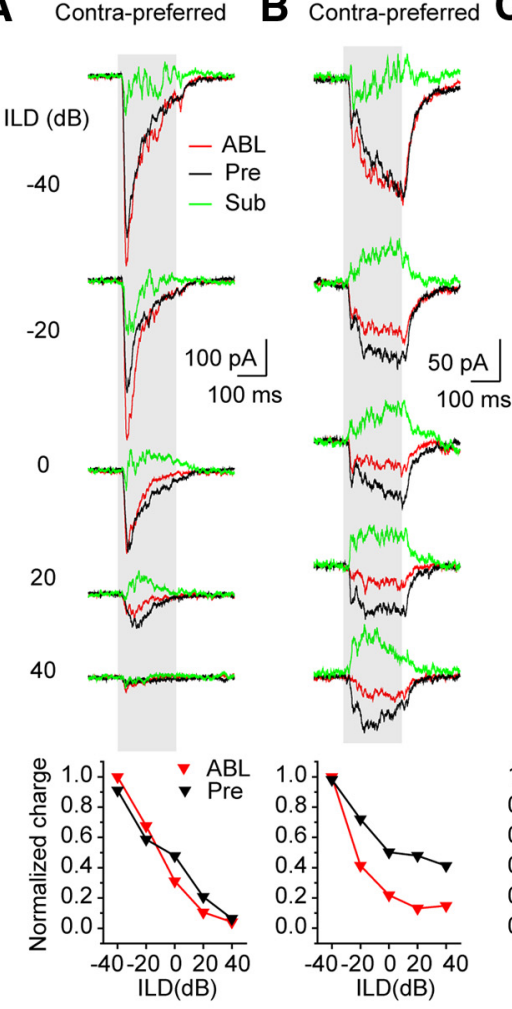

C U-shape

D
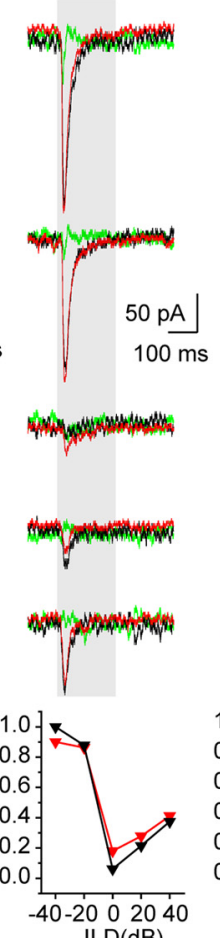

ILD(dB)

$\mathbf{G}_{\text {ePsCs }}$
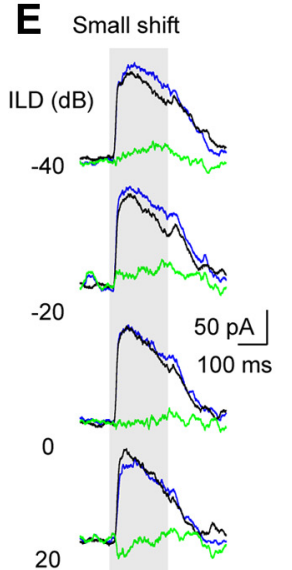

$\mathbf{F}$ Large shift $-\mathrm{ABL}$

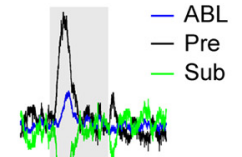

Contra-preferred

- Negative shift

$\mathrm{n}=6$

o Positive shift

$\mathrm{n}=1$

- Average of

filled circles

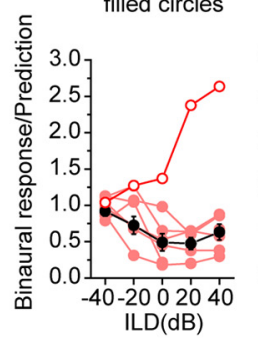

$40 \int_{100}$

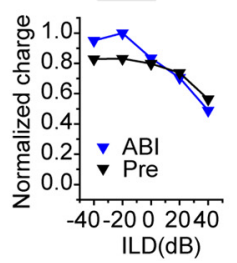

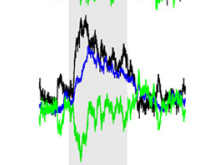

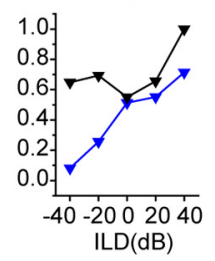

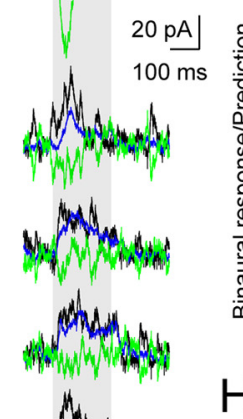

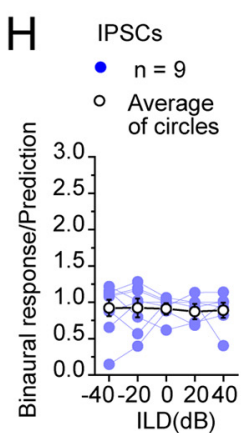

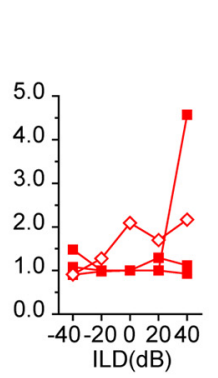
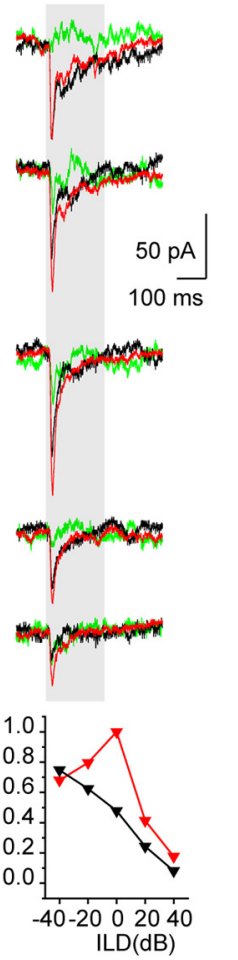

U-shape $=n=3$

Center-preferred $\diamond \mathrm{n}=1$

I

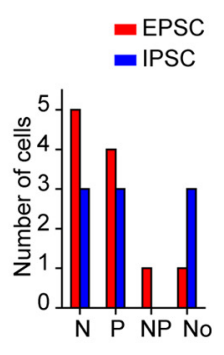

Figure 7. The binaural effects in the responses to ABL stimuli. A-D, EPSCS. E, F, IPSCS. In the top traces, the binaural response (ABL, red lines) and the predicted binaural responses (Pre, black lines) from the monaural response are compared. The subtraction of predicted response from binaural response is presented as a green line (Sub). In the bottom, the normalized charge of binaural and prediction were plotted against ILD. $A, B$, Contra-preferred neurons. C, U-shaped neuron. $\boldsymbol{D}$, Center-preferred neuron. Each response was evoked by $B F$ tone. $B F$ was 17.0, 11.0, 13.0, and $11.0 \mathrm{kHz}$. E, IPSCs with small differences between binaural response and prediction. $\boldsymbol{F}$, IPSCS with a large shift. BF was 11.0 and $10.0 \mathrm{kHz}$. $\boldsymbol{G}, \boldsymbol{H}$, The ratio of the charge of binaural response to that of prediction was plotted against ILD. G, EPSCS. H, IPSCS. I, The types of the shift of binaural responses from prediction. N, Negative shift; P, positive shift; NP, negative and positive shift; №, no shift. The shift >20\% was judged as a significant shift.

Because the ABL stimulus is used to relate ILD coding to sound location on the azimuth, we wondered how the responses to CL-constant and ABL stimuli were related. One consistent relationship was that all the EPSCs of the neurons with center-preferred responses had nonmonotonic enhancement ( $n=4$ of 4 neurons; Fig. $8 C, H$ ). The IPSCs of the neurons with $\mathrm{U}$-shaped responses to $\mathrm{ABL}$ stimuli also consistently showed an enhancement with increasing ipsilateral levels (Fig. $8 F, H$ ). However, the neurons with contra-preferred and U-shaped EPSC responses could show all three patterns to CL-constant stimuli (suppression, enhancement, or nonmonotonic enhancement). Thus, the response to CLconstant stimulus was usually a poor predictor of the response to ABL stimuli.

When neurons had no synaptic currents evoked by monaural ipsilateral stimuli, they usually showed EPSC suppression with the CL-constant stimuli $(n=7$; Fig. $8 H)$. This suggests that their primary input was from a lower binaural structure, such as the LSO. However, there were exceptions. One neuron showed EPSC nonmonotonic enhancement, and two neurons showed no change in EPSCs to CL-constant stimuli (Fig. $8 H)$. This suggests that "pure" monaural neurons were rare in the IC when synaptic inputs are considered and that some ascending binaural inputs may be excitatory in the mouse, possibly from the medial superior olive (MSO).

\section{Binaural: evoked spikes more sharply tuned to ABL stimuli than evoked EPSCs}

In five neurons, we recorded the extracellular spike activity and the synaptic currents in the response to ABL stimuli (Figs. $9,10)$. The small number of cells attests to the difficulty of the experiment because spike recordings were made in cellattached mode before the whole-cell mode was achieved. This procedure was used instead of the easier whole-cell, current-clamp recordings because we used an internal solution that blocked action potentials to make cleaner current recordings in voltage clamp (see Materials and Methods). Moreover, current recordings with high uncompensated series resistance were not included.

We succeeded in obtaining both spikes and current recordings from three neurons with contra-preferred responses to ABL stimuli. Both the spike pattern (Fig. $9 A-C$ ) and the EPSCs (Fig. $9 D-F$ ) of these neurons showed the contra-preferred re- 
sponse. The binaural response curves of the spikes and EPSC (Fig. 9G-I) were highly correlated $(r=0.94,0.94$, and 0.91 for neurons 1,2 , and 3, respectively). This implies that the excitatory inputs were critical in shaping the spike responses. Although the EPSCs and spikes were well correlated, they were not matched completely. For example, in neuron 2, the EPSC at ILD of $20 \mathrm{~dB}$ (Fig. 9E) apparently failed to generate spikes (Fig. 9B, $H$ ). The normalized EPSCs and mean numbers of spike are shown in the insets of Figure $9 G-I$. In these plots, the spike curves showed a steeper slope and were below EPSC curves. These results suggest that the spike response to the ABL stimulus is more sharply tuned along the horizontal azimuth than that of EPSCs.

In neurons 1 and 2, we also succeeded in recording the IPSCs. In these neurons, the EPSCs and IPSCs were highly correlated in the response to ABL stimuli $(r=$ 0.83 and 0.94 for Fig. $9 G$ and $H$, respectively), as was true for the majority of the recorded neurons (Fig. 5E). Thus, balanced inhibition could suppress the excitatory inputs to shape the binaural spike responses to $\mathrm{ABL}$ stimuli (see Discussion).

In two neurons, we did not observed a spike responses to the ABL stimulus (ABL of $40 \mathrm{~dB}$; neuron 4 was tested with five sets of stimuli, and neuron 5 was tested with 10 sets of stimuli). Nevertheless, the evoked synaptic inputs of these neurons were recorded (Fig. 10). In these neurons, the evoked EPSCs were small relative to the IPSCs but well correlated (Fig. 10C,D; $r=0.97$ and 0.91 for neurons 4 and 5, respectively). However, the response of neuron 5 at the ILD of $-40 \mathrm{~dB}$ was unusual. At that ILD, neuron 5 had a transient EPSC whose peak amplitude was larger than those in Figure 9 and earlier than the IPSC. This suggests that the absence of firing in this neuron may also be influenced by nonsynaptic factors, such as resting potential and membrane excitability.

\section{Discussion}

These findings reveal systematic differences in synaptic coding for ILD along a horizontal plane. Highly correlated, temporally matched, monaurally driven EPSCs and IPSCs are found in most IC neurons and contribute to three responses to ABL stimuli: (1) contra-preferred; (2) U-shaped; or (3) center-preferred. In most neurons, the IPSC responses to ABL stimuli are predicted by the monaural responses but less so for EPSCs. Binaural nonlinearities sometimes sharpened the ILD selectivity. Although ILD sensitivity is clearly demonstrated with CL-constant stimuli, those responses were poor predictors of the synaptic response to $\mathrm{ABL}$ stimuli. Finally, the comparison of spike patterns and synaptic currents to ABL stimuli in single neurons showed that they were lateral response.
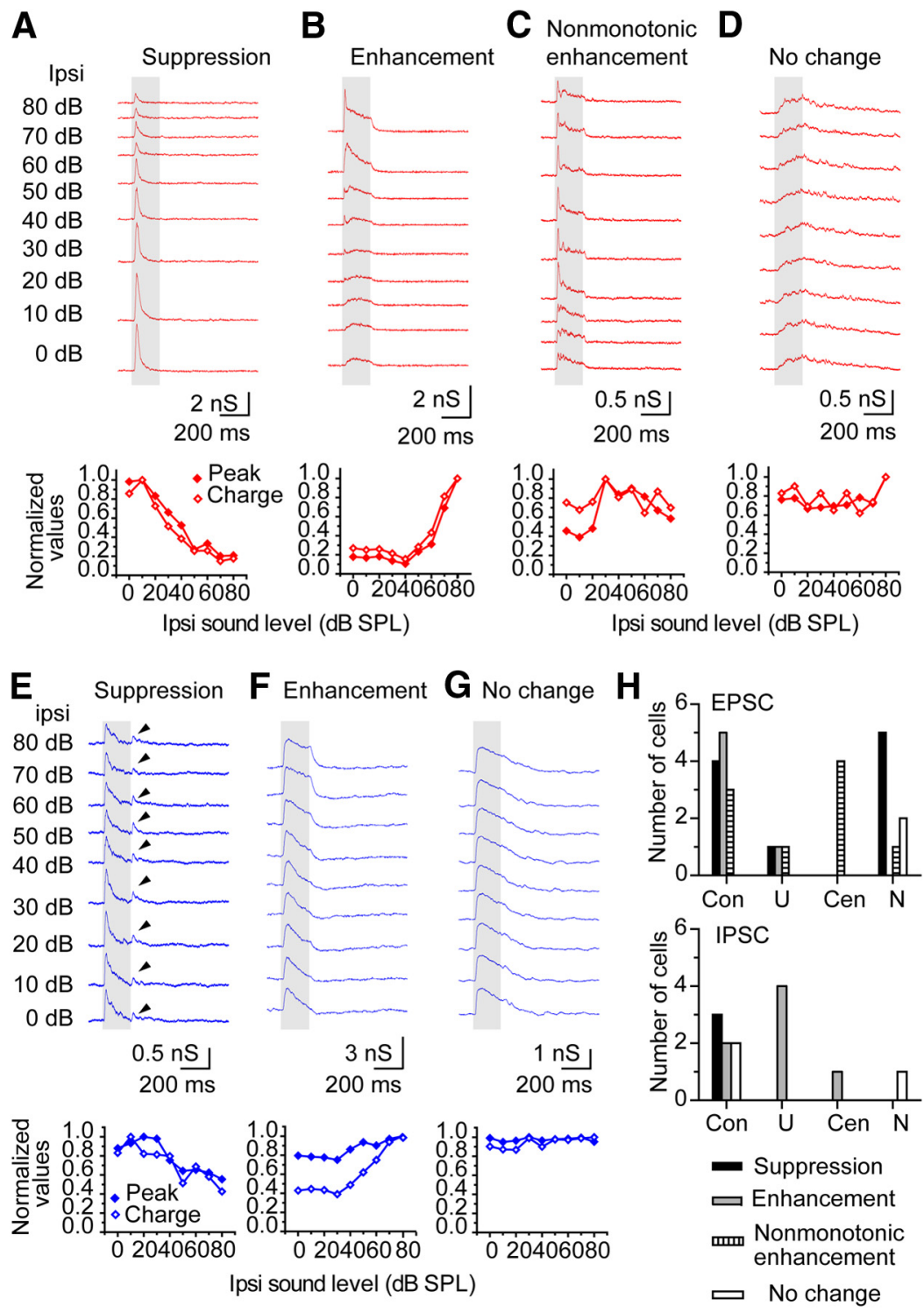

Figure 8. Synaptic responses to $\mathrm{CL}$-constant stimuli. $\boldsymbol{A}-\boldsymbol{D}$, EPSCS. Top traces, The excitatory responses with different ipsilateral sound intensities. $\boldsymbol{A}$, Suppression. $\boldsymbol{B}$, Enhancement. $\boldsymbol{C}$, Nonmonotonic enhancement. $\boldsymbol{D}$, No change. BF tones were used. The BF of , Suppression. $\boldsymbol{F}$, Enhancement. $\boldsymbol{G}$, No change. BF of each neuron was $12.5,10.5$, and $11.0 \mathrm{kHz}$. The sound intensities of contralatral stimuli were 40,40 , and $30 \mathrm{~dB}$. $\boldsymbol{H}$, The population of each type of responses to CL-constant stimuli in the groups classified by the responses to ABL stimuli. Con, Contra-preferred response; $\mathrm{U}, \mathrm{U}$-shaped response; Cen, center-preferred response; $\mathrm{N}$, no ipsi-

well correlated, but the spike response had sharper ILD tuning than the synaptic response. In summary, balanced excitatory and inhibitory inputs may be a general feature of synaptic coding for many types of sound processing in the IC.

\section{Previous studies of synaptic responses to ILD}

Two recent studies investigated ILD processing in the IC using whole-cell in vivo recordings but emphasize different aspects (Li and Pollak, 2013; Xiong et al., 2013). The difficulty of these experiments is obvious from the small numbers of neurons reported $(n=10$ and 18, respectively). Li and Pollak (2013) offer significant insights on the circuitry that underlies ILD coding by EI neurons (excited by the contralateral ear; inhibited by the ipsilateral ear). Xiong et al. (2013) investigated the frequency tuning of IC neurons to show gain control and synaptic scaling during ILD processing. 
A

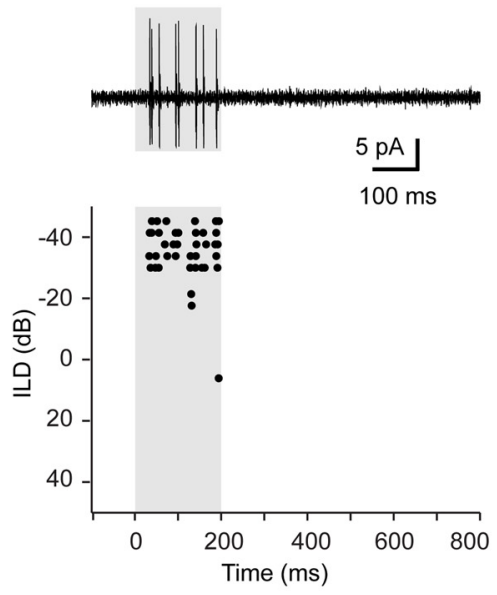

D

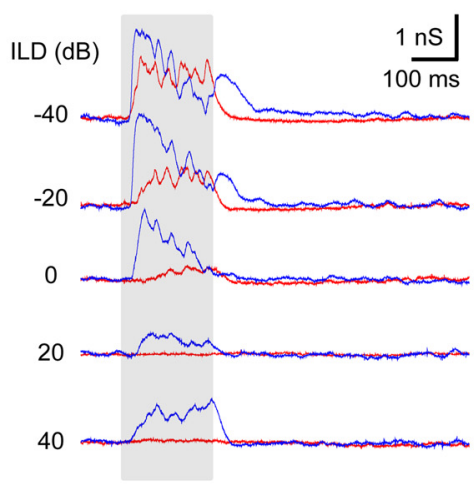

G
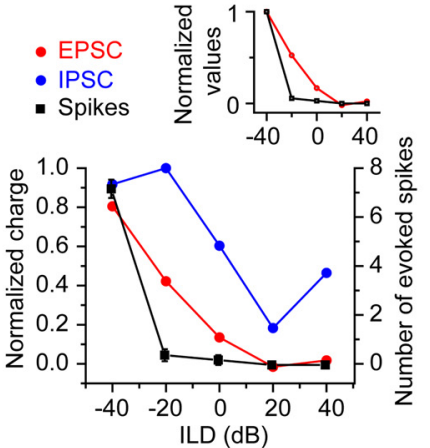

B
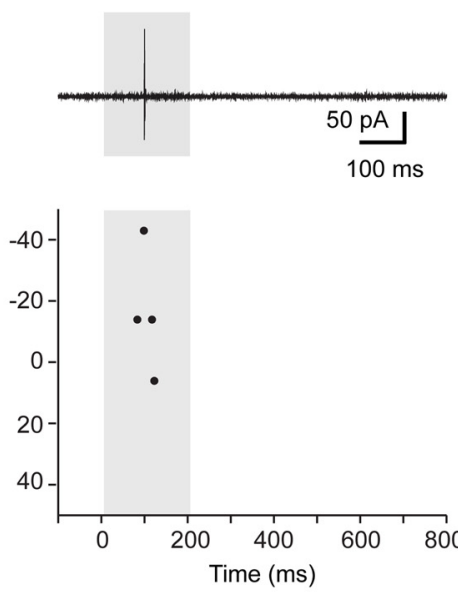

E

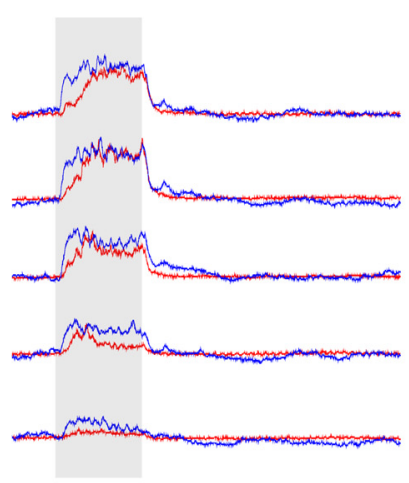

H
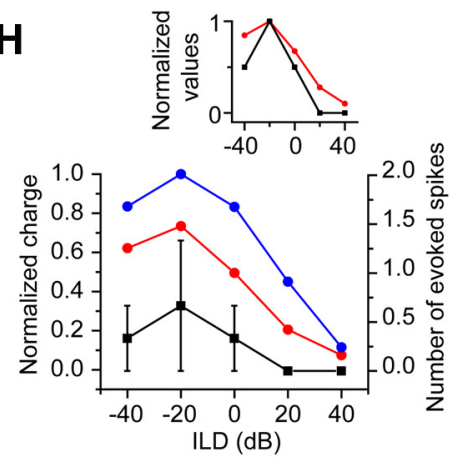

C

Neuron 3
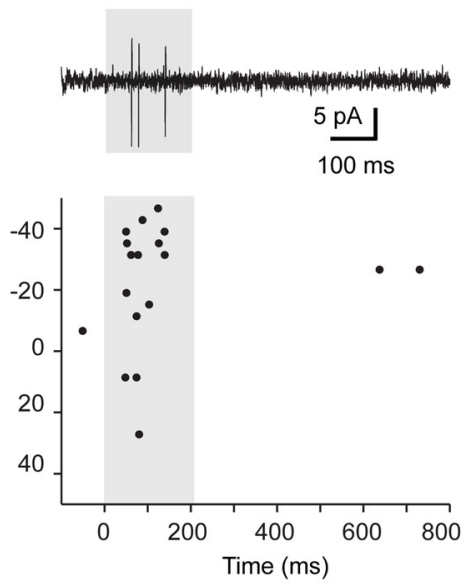

F

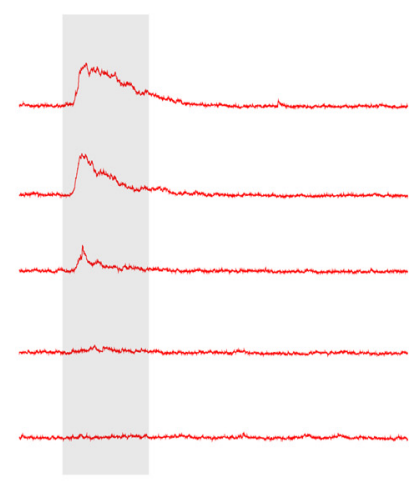

I
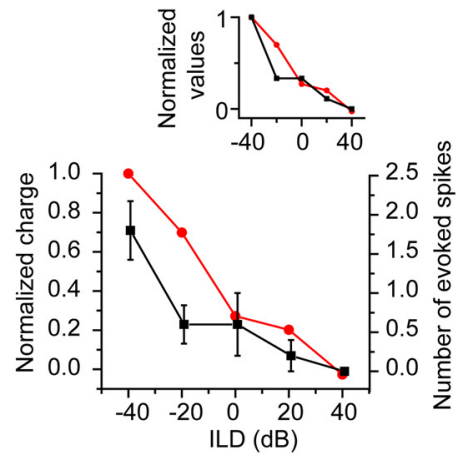

Figure 9. The evoked spikes and PSCS to ABL stimuli. The recordings from three neurons were presented $(\boldsymbol{A}, \boldsymbol{D}, \boldsymbol{G}$ from neuron $1 ; \boldsymbol{B}, \boldsymbol{E}, \boldsymbol{H}$ from neuron $2 ; \boldsymbol{C}, \boldsymbol{F}, \mathbf{I}$ from neuron 3$) . \boldsymbol{A}-\boldsymbol{C}$, The spike responses to $A B L$ stimuli. The ABL was $40 \mathrm{~dB}$. Neurons $1(\boldsymbol{A})$ and $2(\boldsymbol{B})$ were tested by white noise. Neuron 3 was tested by BF tones $(18 \mathrm{kHz})$. Neurons 1 and 3 were tested by five sets of stimuli, and neuron 2 was tested by three sets of stimuli. The top traces were current responses to the sound at an ILD of $-40 \mathrm{~dB}$. The bottom panels were the raster plots to ABL stimuli. $D-F$, The evoked synaptic inputs to ABL stimuli. Red, EPSCs. Blue, IPSCs. G-I, The response curves of the normalized synaptic inputs (red, EPSC; blue, IPSCs) and spike responses (black) to ABL stimuli. The left $y$-axis is for normalized charge, and the right $y$-axis is for number of evoked spikes. In the insets, the charge of EPSC and mean spike numbers are normalized and plotted.

Our study differs from that of Li and Pollak (2013) in terms of the species, sampling method, acoustic stimulus, and recording method. They sampled only the EI neurons in the IC of the Mexican free-tailed bat and used the CL-constant protocol with 5-20 ms tones. Conductances were modeled from current-clamp recordings. They discovered an unexpected excitatory synaptic response to ipsilateral monaural stimuli. Despite different circuitry, they conclude that all EI cells receive an input from the LSO based on either a lack of IPSPs or a binaural conductance that was smaller than the monaural conductance. In contrast, the present findings show that some, but not all, neurons show a suppression of binaural EPSCs by higher levels of ipsilateral stimulation. We also find enhancement and nonmonotonic enhancement responses attributable to an ipsilateral excitatory monotonic or nonmonotonic input. Moreover, the response to CL-constant stimuli poorly predicted the response to ABL stimuli.

Although methodologically similar to the present study, Xiong et al. (2013) studied spectral responses under a limited set of binaural conditions. In most neurons, they used only ILD of 0 for calculating aural dominance based on the amplitudes of EPSCs or IPSCs summed across frequency. When they tested four different ILD levels in seven neurons, they conclude that ILD-level gain modulation is achieved by modulating only the excitatory input. This result is inconsistent with our larger sample 
of ILD-sensitive neurons studied with the ABL stimuli in which we find the excitatory and inhibitory inputs to be highly correlated and, thus, well balanced in most neurons. However, it agrees with our finding that in some neurons the response to binaural ABL stimuli may be suppressed relative to the predicted monaural response when the ipsilateral ear is more intense.

\section{Bilateral matched responses suggest a binaural comparator}

Commissural neurons may link neurons with similar properties in IC. One surprising result was that the temporal properties of the contralateral and ipsilateral EPSCs were well matched. This temporal matching was despite EPSCs with diverse temporal patterns and was observed across many neurons with diverse peak times. This finding suggests that the same ascending sources may drive commissurally connected neurons. Commissural neurons send a dominantly excitatory projection to the contralateral IC that connects symmetrical locations in the IC on both sides (Saldaña and Merchán, 1992; Hernández et al., 2006). This input might be common in most neurons and implies that microcircuits with similar functions are matched and connected on both sides of the IC.

Coding of sound location in space also may require a binaural comparator (Palmer and Kuwada, 2005; Grothe et al., 2010). This mechanism may be supplied by the convergence of excitatory inputs from both ears via the IC commissure. Binaural comparisons may be made for both interaural time differences (ITDs) and ILDs because ITDs may be coded by the envelope of the sound at high frequencies (Fitzpatrick et al., 2000; Bernstein and Trahiotis, 2002). Thus, the neurons with bilateral EPSCs in the mouse could compare both ILDs and ITDs.

\section{Multiple mechanisms for ILD-based sound localization}

The present results with ABL stimuli are consistent with previous extracellular recordings in the IC of cats and gerbils (Irvine, 1987; Semple and Kitzes, 1987; Irvine and Gago, 1990). They showed that the contra-preferred response was the most common, but the center-preferred response was the second-most common. Our results showed that the responses to ABL stimuli substantially reflect the contralateral and ipsilateral monaural responses. This is consistent with previous extracellular studies with virtual auditory
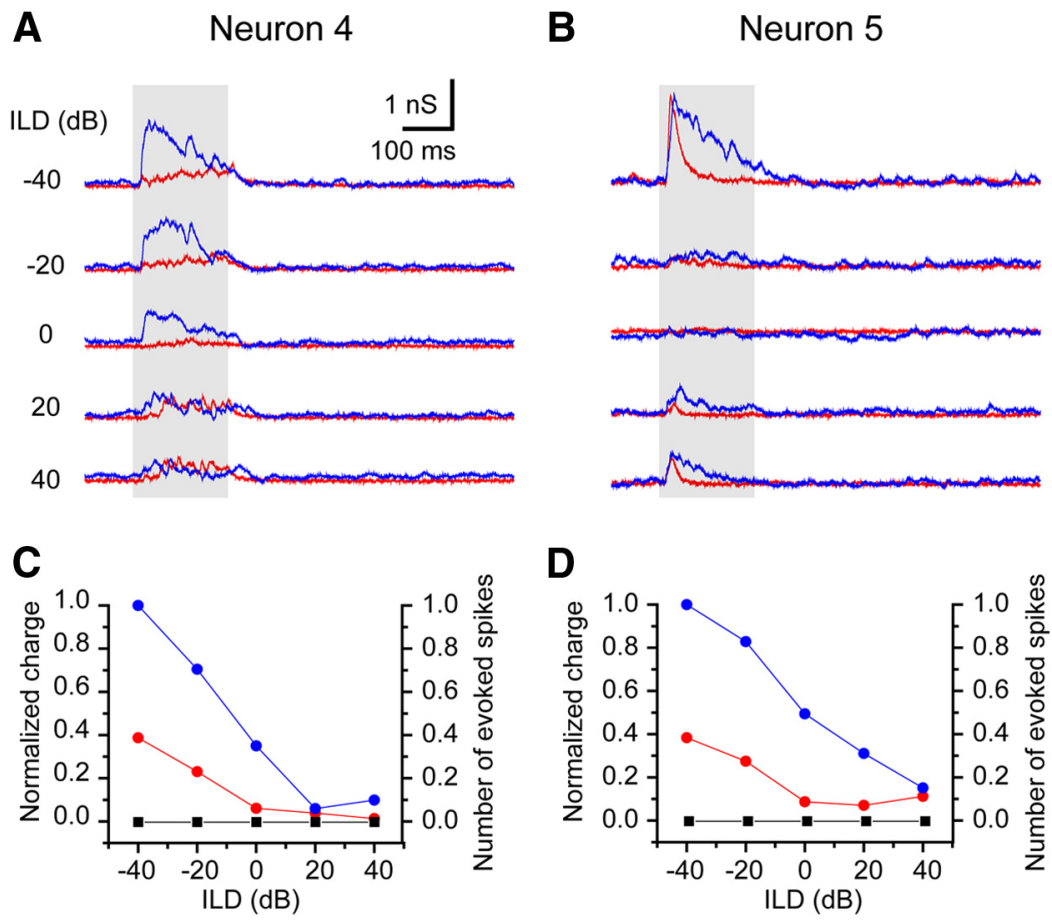

Figure 10. The neurons with no evoked spikes to $40 \mathrm{~dB} A B L$ stimuli. The recordings from two neurons are presented $(\boldsymbol{A}, \boldsymbol{C}$ from neuron $4 ; \boldsymbol{B}, \boldsymbol{D}$ from neuron 5). Both neurons were tested by white noise. $\boldsymbol{A}, \boldsymbol{B}$, The evoked synaptic inputs to $A B L$ stimuli. Red, EPSC; ; blue, IPSCS. C, D, The response curves of the normalized synaptic inputs (red, EPSC; blue, IPSCS) and spike responses (black) to ABL stimuli. The left $y$-axis is for normalized charge, and the right $y$-axis is for number of evoked spikes.

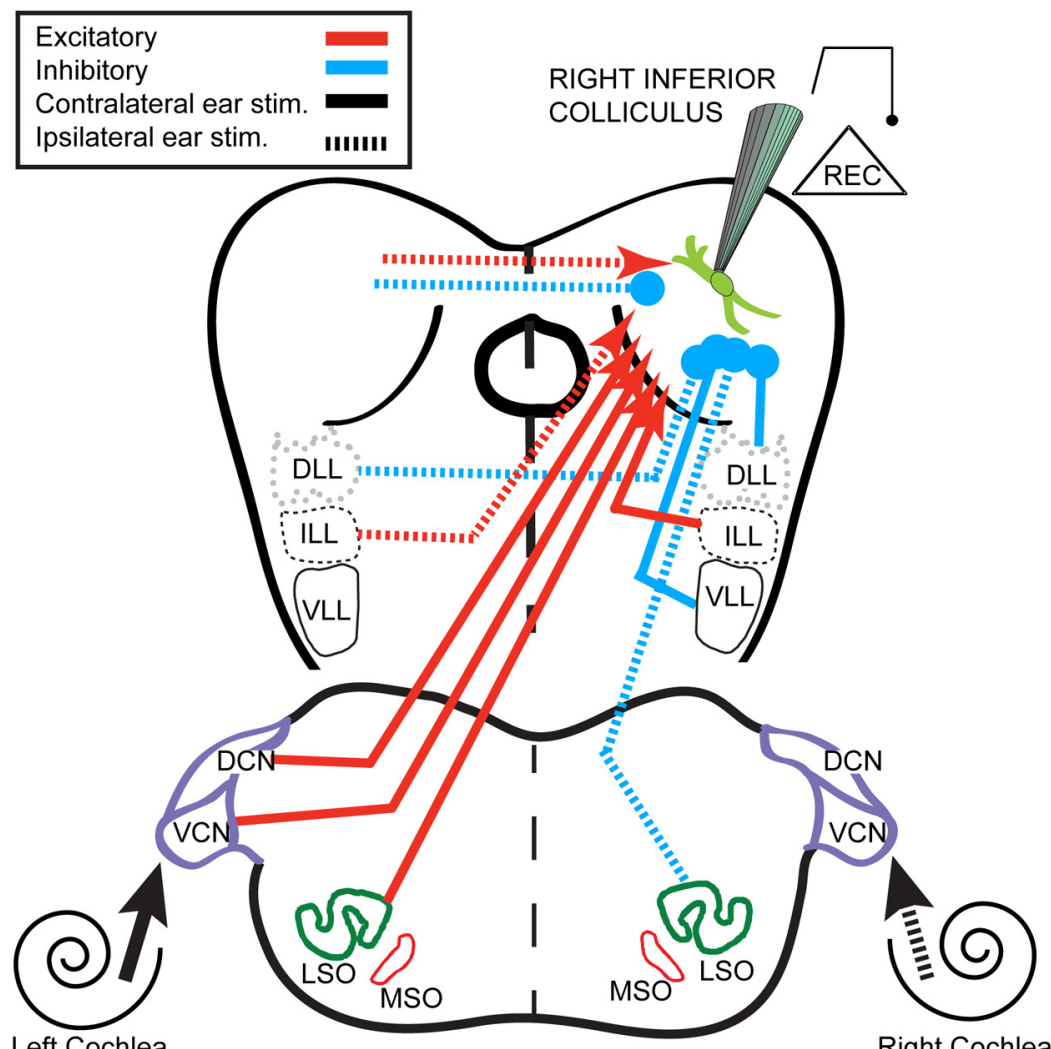

Right Cochlea

Figure 11. Depiction of possible inputs to the IC. The schematic drawing of the auditory neural pathways to IC. Red lines indicate the excitatory pathways. Blue lines indicate the inhibitory pathways. Solid lines indicate the contralaterally driven pathways, and dotted lines indicate the ipsilaterally driven pathways. The abbreviations of the auditory nuclei are shown in the figure. VCN, Ventral cochlear nucleus; DCN, dorsal cochlear nucleus; DLL, dorsal lateral lemniscus; VLL, ventral lateral lemniscus; ILL, intermediate lateral lemniscus. 
stimuli in which the contralateral monaural response primarily shapes azimuthal tuning at low stimulus levels (Delgutte et al., 1999; Kuwada et al., 2011).

Excitatory ascending pathways may be more nonlinear and result from a combination of monaural and binaural sources (Fig. 11). The monaural EPSCs driven contralaterally arise from the cochlear nucleus, but the ipsilaterally driven EPSCs might be attributable to the commissural input from the contralateral IC (Fig. 11; Malmierca et al., 2009). Direct excitatory inputs from the ipsilateral cochlear nucleus are absent in rodents and bats (Oliver et al., 1999; Hernández et al., 2006; Li and Pollak, 2013). Descending excitation from the neocortex is possible but inconsistent with the observed short latencies. Ipsilateral monaural excitation via the MSO is possible but unlikely because of its small size in mice. Binaural inputs to the IC from the LSO are consistent with contralateral monaural stimulation evoking larger EPSCs than binaural stimulation (Guinan et al., 1972; Tsuchitani, 1977). Thus, binaural suppression in contra-preferred neurons may be attributable to reduced inputs from the LSO (Li et al., 2010; Li and Pollak, 2013). In contrast, binaural nonmonotonic enhancement was seen in the center-preferred neurons and is, perhaps, attributable to binaural inputs from the MSO (Irving and Harrison, 1967). However, the superior olive is not necessary for ILD sensitivity in the IC (Li and Kelly, 1992). Unusual, bilateral, excitatory inputs to the IC from the intermediate nucleus of the lateral lemniscus (NLL) in mouse (Frisina et al., 1998; Ito et al., 2011) may create binaural enhancement de novo and shape the U-shaped response.

Inhibitory ascending pathways may be more "linear" than the excitatory pathways because they show less binaural suppression or enhancement regardless of the binaural response type to $\mathrm{ABL}$ stimuli. The IPSCs to ABL stimuli were well predicted by the sum of the monaural IPSCs. Although the IC has numerous inhibitory inputs (Fig. 11; Malmierca and Hackett, 2010), monaural inputs from the ventral NLL are most prominent (Saint Marie and Baker, 1990; Batra and Fitzpatrick, 2002). Perhaps these dominate binaural inhibitory inputs from the LSO and the dorsal NLL (Bajo et al., 1998; Kelly et al., 1998). IPSC driven by an ipsilateral stimulus may be from the ipsilateral LSO (Fig. 11; Oliver et al., 1995) or contralateral dorsal NLL (Li and Kelly, 1992; Burger and Pollak, 2001).

\section{Balanced EPSCs and IPSCs as a general mode of synaptic activation in IC}

Our data show that the balance of excitation and inhibition may be an important general mechanism for neural activation in the IC in ILDs and other types of sound processing. In most neurons, EPSCs and IPSCs were highly correlated. Frequency-matched, balanced inhibition may regulate the gain of the neural response and prevent small EPSCs from generating spikes (Palombi and Caspary, 1996). Indeed, it appears to be essential to improve the dynamic range in sound level coding in the IC (Sivaramakrishnan et al., 2004). This suppression of excitation is also suggested in the neurons in which both spikes and synaptic responses were recorded because the ILD tuning curve with spikes was sharper than that with EPSCs. Uncorrelated excitatory and inhibitory inputs were seen here only in center-preferred neurons and resemble other reports of IC neurons with unbalanced excitatory and inhibitory synaptic responses (Kuo and Wu, 2012; Xiong et al., 2013).

The inputs and circuitry of the IC are well designed to provide a balanced excitatory and inhibitory input to the postsynaptic neurons. Although balanced excitation and inhibition has been observed in the cerebral cortex (Borg-Graham et al., 1998; Wehr and Zador, 2003), the cortical circuitry differs from that of the IC because of inhibition arises from local GABAergic interneurons driven by ascending or cortical excitation. In contrast, IC neurons receive parallel excitatory and inhibitory inputs from the lower auditory brainstem that include substantial, direct GABAergic and glycinergic inhibitory inputs. These parallel inputs form layers that engulf disc-shaped IC neurons. Thus, all IC neurons will receive at least one direct excitatory and one direct inhibitory input (Loftus et al., 2010) that will provide temporally synchronous, balanced excitatory and inhibitory synaptic inputs to the IC neuron.

\section{References}

Allen PD, Ison JR (2010) Sensitivity of the mouse to changes in azimuthal sound location: angular separation, spectral composition, and sound level. Behav Neurosci 124:265-277. CrossRef Medline

Bajo VM, Villa AE, de Ribaupierre F, Rouiller EM (1998) Discharge properties of single neurons in the dorsal nucleus of the lateral lemniscus of the rat. Brain Res Bull 47:595-610. CrossRef Medline

Batra R, Fitzpatrick DC (2002) Monaural and binaural processing in the ventral nucleus of the lateral lemniscus: a major source of inhibition to the inferior colliculus. Hear Res 168:90-97. CrossRef Medline

Bernstein LR, Trahiotis C (2002) Enhancing sensitivity to interaural delays at high frequencies by using "transposed stimuli." J Acoust Soc Am 112: 1026-1036. CrossRef

Borg-Graham LJ, Monier C, Frégnac Y (1998) Visual input evokes transient and strong shunting inhibition in visual cortical neurons. Nature 393: 369-373. CrossRef Medline

Burger RM, Pollak GD (2001) Reversible inactivation of the dorsal nucleus of the lateral lemniscus reveals its role in the processing of multiple sound sources in the inferior colliculus of bats. J Neurosci 21:4830-4843. Medline

Caspary DM, Raza A, Lawhorn Armour BA, Pippin J, Arnerić SP (1990) Immunocytochemical and neurochemical evidence for age-related loss of GABA in the inferior colliculus: implications for neural presbycusis. J Neurosci 10:2363-2372. Medline

Dallos P (1973) The auditory periphery. New York: Academic.

Delgutte B, Joris PX, Litovsky RY, Yin TC (1999) Receptive fields and binaural interactions for virtual-space stimuli in the cat inferior colliculus. J Neurophysiol 81:2833-2851. Medline

Fitzpatrick DC, Kuwada S, Batra R (2000) Neural sensitivity to interaural time differences: beyond the Jeffress model. J Neurosci 20:1605-1615. Medline

Frisina RD, Walton JP, Lynch-Armour MA, Byrd JD (1998) Inputs to a physiologically characterized region of the inferior colliculus of the young adult CBA mouse. Hear Res 115:61-81. CrossRef Medline

Grothe B, Pecka M, McAlpine D (2010) Mechanisms of sound localization in mammals. Physiol Rev 90:983-1012. CrossRef Medline

Guinan JJ, Guinan SS, Norris BE (1972) Single auditory units in the superior olivary complex I: responses to sounds and classifications based on physiological properties. Int J Neurosci 4:101-120. CrossRef

Hernández O, Rees A, Malmierca MS (2006) A GABAergic component in the commissure of the inferior colliculus in rat. Neuroreport 17:16111614. CrossRef Medline

Irvine DR (1987) A comparison of two methods for the measurement of neural sensitivity to interaural intensity differences. Hear Res 30:169179. CrossRef Medline

Irvine DR, Gago G (1990) Binaural interaction in high-frequency neurons in inferior colliculus of the cat: effects of variations in sound pressure level on sensitivity to interaural intensity differences. J Neurophysiol 63:570591. Medline

Irving R, Harrison JM (1967) The superior olivary complex and audition: a comparative study. J Comp Neurol 130:77-86. CrossRef Medline

Ito T, Oliver DL (2010) Origins of glutamatergic terminals in the inferior colliculus identified by retrograde transport and expression of VGLUT1 and VGLUT2 genes. Front Neuroanat 4:135. CrossRef Medline

Ito T, Bishop DC, Oliver DL (2011) Expression of glutamate and inhibitory amino acid vesicular transporters in the rodent auditory brainstem. J Comp Neurol 519:316-340. CrossRef Medline

Kelly JB, Buckthought AD, Kidd SA (1998) Monaural and binaural response 
properties of single neurons in the rat's dorsal nucleus of the lateral lemniscus. Hear Res 122:25-40. CrossRef Medline

Kuo RI, Wu GK (2012) The generation of direction selectivity in the auditory system. Neuron 73:1016-1027. CrossRef Medline

Kuwada S, Batra R, Yin TC, Oliver DL, Haberly LB, Stanford TR (1997) Intracellular recordings in response to monaural and binaural stimulation of neurons in the inferior colliculus of the cat. J Neurosci 17:75657581. Medline

Kuwada S, Bishop B, Alex C, Condit DW, Kim DO (2011) Spatial tuning to sound-source azimuth in the inferior colliculus of unanesthetized rabbit. J Neurophysiol 106:2698-2708. CrossRef Medline

Lauer AM, Slee SJ, May BJ (2011) Acoustic basis of directional acuity in laboratory mice. J Assoc Res Otolaryngol 12:633-645. CrossRef Medline

Li L, Kelly JB (1992) Binaural responses in rat inferior colliculus following kainic acid lesions of the superior olive: interaural intensity difference functions. Hear Res 61:73-85. CrossRef Medline

Li N, Pollak GD (2013) Circuits that innervate excitatory-inhibitory cells in the inferior colliculus obtained with in vivo whole cell recordings. J Neurosci 33:6367-6379. CrossRef Medline

Li N, Gittelman JX, Pollak GD (2010) Intracellular recordings reveal novel features of neurons that code interaural intensity disparities in the inferior colliculus. J Neurosci 30:14573-14584. CrossRef Medline

Loftus WC, Bishop DC, Oliver DL (2010) Differential patterns of inputs create functional zones in central nucleus of inferior colliculus. J Neurosci 30:13396-13408. CrossRef Medline

Malmierca MS, Hackett TA (2010) Structural organization of the ascending auditory pathway. In: The oxford handbook of auditory science: the auditory brain (Rees A, Palmer AR, eds), pp 9-42. Oxford: Oxford UP.

Malmierca MS, Hernández O, Antunes FM, Rees A (2009) Divergent and point-to-point connections in the commissural pathway between the inferior colliculi. J Comp Neurol 514:226-239. CrossRef Medline

Margrie TW, Brecht M, Sakmann B (2002) In vivo, low-resistance, wholecell recordings from neurons in the anaesthetized and awake mammalian brain. Pflugers Arch 444:491-498. CrossRef Medline

Oliver DL (2000) Ascending efferent projections of the superior olivary complex. Microsc Res Tech 51:355-363. CrossRef Medline

Oliver DL, Beckius GE, Shneiderman A (1995) Axonal projections from the lateral and medial superior olive to the inferior colliculus of the cat: a study using electron microscopic autoradiography. J Comp Neurol 360: 17-32. CrossRef Medline
Oliver DL, Ostapoff EM, Beckius GE (1999) Direct innervation of identified tectothalamic neurons in the inferior colliculus by axons from the cochlear nucleus. Neuroscience 93:643-658. CrossRef Medline

Ono M, Yanagawa Y, Koyano K (2005) GABAergic neurons in inferior colliculus of the GAD67-GFP knock-in mouse: electrophysiological and morphological properties. Neurosci Res 51:475-492. CrossRef Medline

Palmer AR, Kuwada S (2005) Binaural spatial coding in the inferior colliculus. In: The inferior colliculus (Winer JA, Schreiner CE, eds), pp 377-410. New York: Springer.

Palombi PS, Caspary DM (1996) GABA inputs control discharge rate primarily within frequency receptive fields of inferior colliculus neurons. J Neurophysiol 75:2211-2219. Medline

Park TJ, Pollak GD (1994) Azimuthal receptive fields are shaped by GABAergic inhibition in the inferior colliculus of the mustache bat. J Neurophysiol 72:1080-1102. Medline

Saint Marie RL, Baker RA (1990) Neurotransmitter-specific uptake and retrograde transport of $\left[{ }^{3} \mathrm{H}\right]$ glycine from the inferior colliculus by ipsilateral projections of the superior olivary complex and nuclei of the lateral lemniscus. Brain Res 524:244-253. CrossRef Medline

Saldaña E, Merchán MA (1992) Intrinsic and commissural connections of the rat inferior colliculus. J Comp Neurol 319:417-437. CrossRef Medline

Semple MN, Kitzes LM (1987) Binaural processing of sound pressure level in the inferior colliculus. J Neurophysiol 57:1130-1147. Medline

Sivaramakrishnan S, Sterbing-D'Angelo SJ, Filipovic B, D'Angelo WR, Oliver DL, Kuwada S (2004) $\mathrm{GABA}_{\mathrm{A}}$ synapses shape neuronal responses to sound intensity in the inferior colliculus. J Neurosci 24:5031-5043. CrossRef Medline

Sun YJ, Wu GK, Liu BH, Li P, Zhou M, Xiao Z, Tao HW, Zhang LI (2010) Fine-tuning of pre-balanced excitation and inhibition during auditory cortical development. Nature 465:927-931. CrossRef Medline

Tsuchitani C (1977) Functional organization of lateral cell groups of cat superior olivary complex. J Neurophysiol 40:296-318. Medline

Wehr M, Zador AM (2003) Balanced inhibition underlies tuning and sharpens spike timing in auditory cortex. Nature 426:442-446. CrossRef Medline

Xiong XR, Liang F, Li H, Mesik L, Zhang KK, Polley DB, Tao HW, Xiao Z, Zhang LI (2013) Interaural level difference-dependent gain control and synaptic scaling underlying binaural computation. Neuron 79:738-753. CrossRef Medline 